Prepared in cooperation with the Federal Emergency Management Agency

\title{
Monitoring Storm Tide and Flooding from Hurricane Sandy along the Atlantic Coast of the United States, October 2012
}
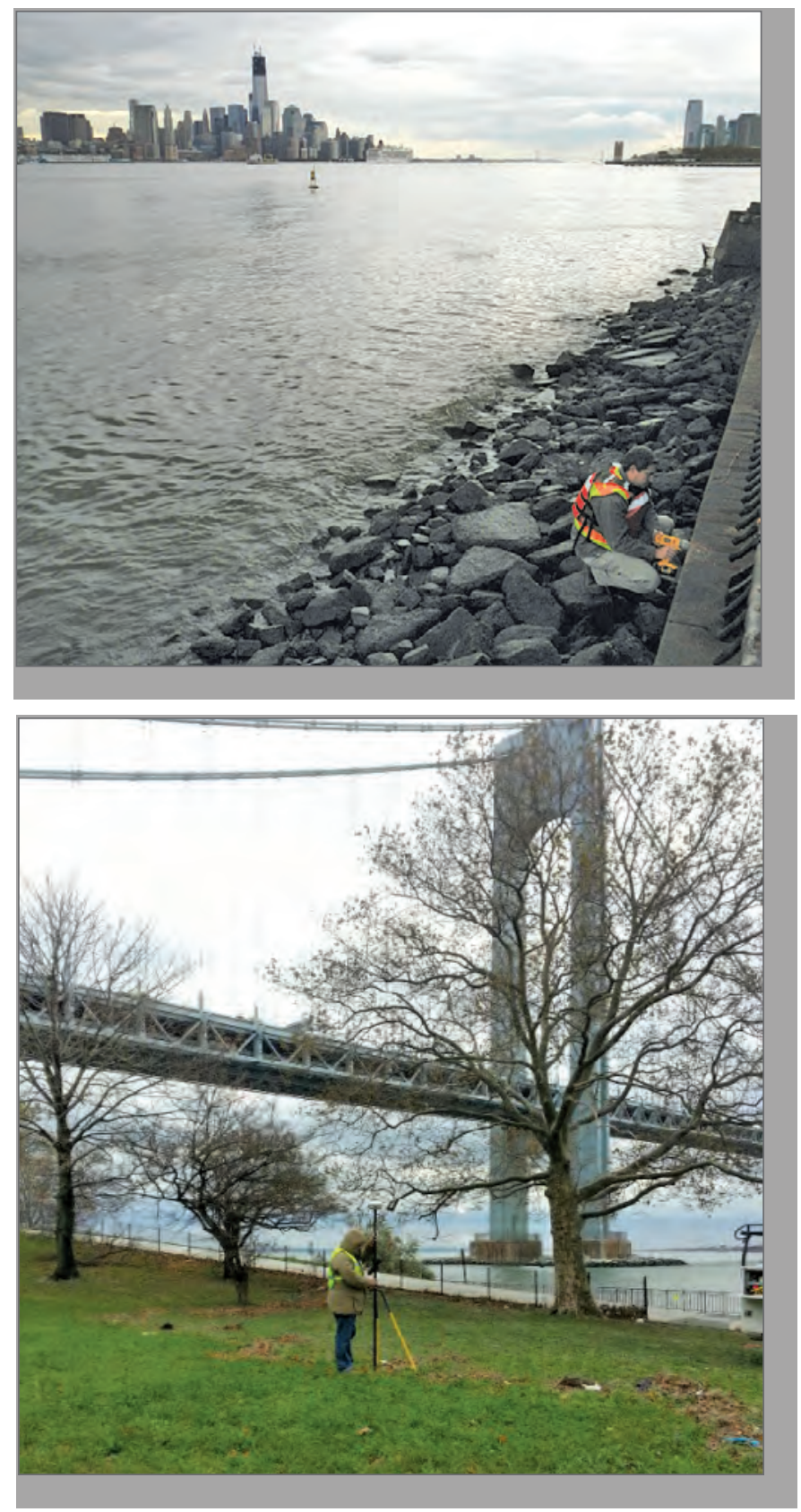

Open-File Report 2013-1043

U.S. Department of the Interior

U.S. Geological Survey
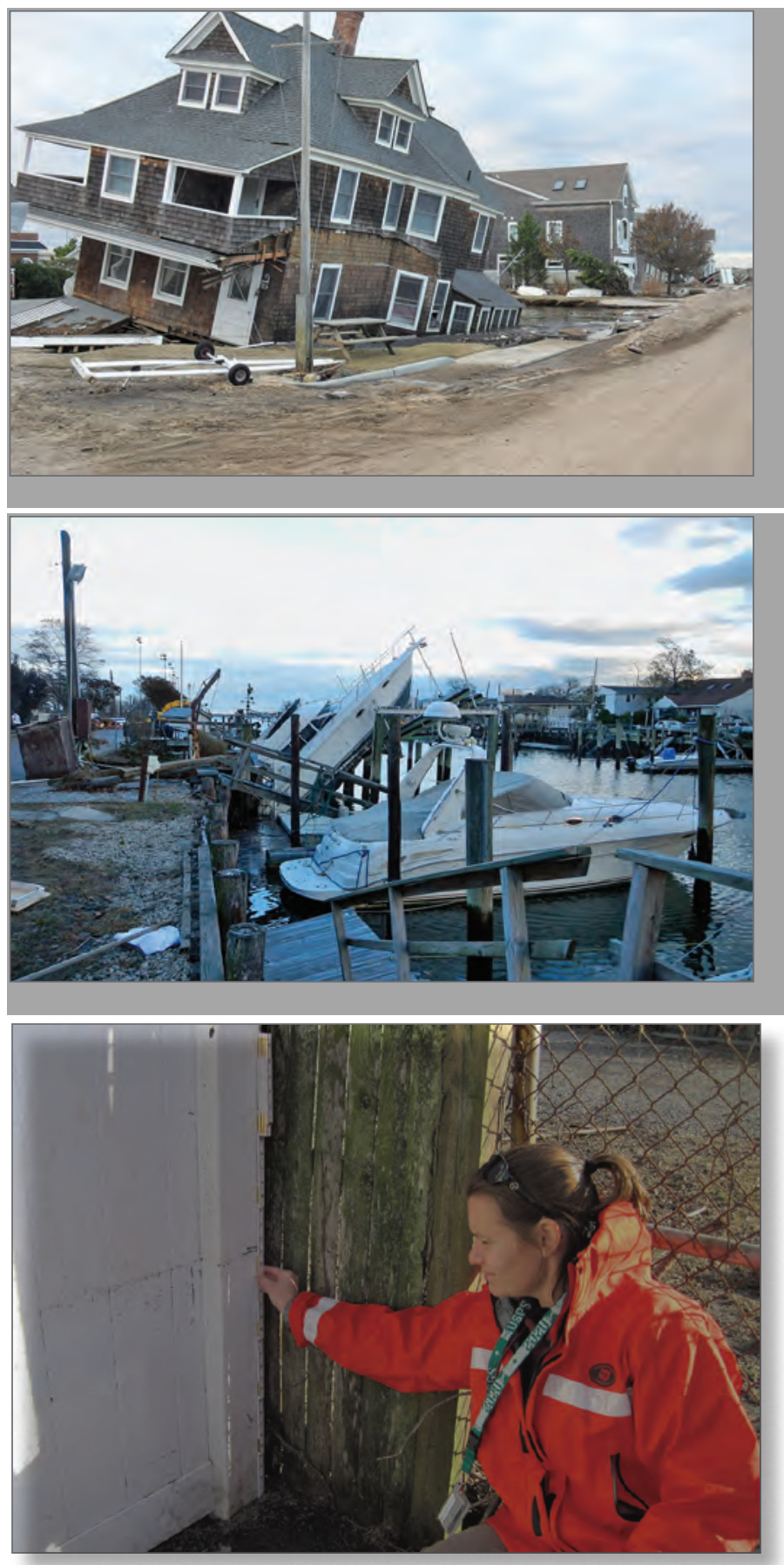


\section{Cover photographs.}

Top left: USGS technician recovering a storm-tide water-level sensor along the Hudson River in Hoboken, New Jersey (photo by Kerry A. Caslow, USGS).

Bottom left: USGS scientist surveying storm-tide debris line near the Verrazano-Narrows Bridge in Kings County, New York (photo by Christopher J. Henry, USGS).

Top right: House in Mantoloking, New Jersey, damaged by Hurricane Sandy (photo by Christopher A. Smith, USGS).

Middle right: Boat in New Jersey damaged by Hurricane Sandy (photo by Christopher J. Henry, USGS).

Bottom right: USGS technician measuring a high-water mark in Suffolk County, New York (photo by Martyn J. Smith, USGS). 


\section{Monitoring Storm Tide and Flooding from Hurricane Sandy along the Atlantic Coast of the United States, October 2012}

By Brian E. McCallum, Shaun M. Wicklein, Robert G. Reiser, Ronald Busciolano, Jonathan Morrison, Richard J. Verdi, Jaime A. Painter, Eric R. Frantz, and Anthony J. Gotvald

Prepared under a mission assignment with the Federal Emergency Management Agency

Open-File Report 2013-1043 


\title{
U.S. Department of the Interior \\ KEN SALAZAR, Secretary
}

\author{
U.S. Geological Survey \\ Suzette M. Kimball, Acting Director
}

\author{
U.S. Geological Survey, Reston, Virginia: 2013
}

For more information on the USGS—-the Federal source for science about the Earth, its natural and living resources,
natural hazards, and the environment, visit http://wWw. usgs.gov or call 1-888-ASK-USGS
For an overview of USGS information products, including maps, imagery, and publications,
visit http://www.usgs.gov/pubprod
To order this and other USGS information products, visit http://store.usgs.gov

Any use of trade, product, or firm names is for descriptive purposes only and does not imply endorsement by the U.S. Government.

Although this report is in the public domain, permission must be secured from the individual copyright owners to reproduce any copyrighted materials contained within this report.

Suggested citation:

McCallum, B.E., Wicklein, S.M., Reiser, R.G., Busciolano, Ronald, Morrison, Jonathan, Verdi, R.J., Painter, J.A., Frantz, E.R., and Gotvald, A.J., 2013, Monitoring storm tide and flooding from Hurricane Sandy along the Atlantic coast of the United States, October 2012: U.S. Geological Survey Open-File Report 2013-1043, 42 p., http://pubs.usgs.gov/of/2013/1043/. 


\title{
Monitoring Storm Tide and Flooding from Hurricane Sandy along the Atlantic Coast of the United States, October 2012
}

\author{
By Brian E. McCallum, Shaun M. Wicklein, Robert G. Reiser, Ronald Busciolano, Jonathan Morrison, \\ Richard J. Verdi, Jaime A. Painter, Eric R. Frantz, and Anthony J. Gotvald
}

\section{Introduction}

The U.S. Geological Survey (USGS) deployed a temporary monitoring network of water-level and barometric pressure sensors at 224 locations along the Atlantic coast from Virginia to Maine to continuously record the timing, areal extent, and magnitude of hurricane storm tide and coastal flooding generated by Hurricane Sandy (fig. 1). These records were greatly supplemented by an extensive post-flood high-water mark (HWM) flagging and surveying campaign from November to December 2012 involving more than 950 HWMs. Both efforts were undertaken as part of a coordinated federal emergency response as outlined by the Stafford Act under a directed mission assignment by the Federal Emergency Management Agency (FEMA).

\section{Hurricane Sandy Storm-Tide Monitoring}

Hurricane Sandy approached the coast of New Jersey near Atlantic City on October 29, 2012, as a Category 1 hurricane on the Saffir-Simpson Hurricane Wind Scale (National Weather Service, 1974). The large size of the storm created a wind field along to the coast that posed significant storm tide impacts. Storm tide, as defined by the National Oceanic and Atmospheric Administration (NOAA; National Oceanic and Atmospheric Administration, 2008), is the water-level rise generated by a combination of storm surge and astronomical tide during a coastal storm.

Given the historic magnitude of the storm and the expected scale of the resulting econonic damages and human hardship, and likely need for timely flood information on which to base both recovery operations and rebuilding efforts,
FEMA mission assigned the USGS to deploy storm-tide monitoring instruments to characterize the height, extent, and timing of storm tides better than could be accomplished by existing USGS or NOAA observational fixed-place networks. In response, a total of 162 water-level and wave-height sensors were deployed at 147 locations during October 26-29 prior to landfall. This represented the second largest deployment of storm-tide sensors, behind only Hurricane Irene which made landfall in the same vicinity in August 2011. To supplement the records provided by these instruments, FEMA also mission-assigned the USGS to conduct an extensive HWM campaign. The resulting database of $950 \mathrm{HWMs}$ following Sandy was the single largest HWM recovery effort in recent USGS history. During and after the storm, data from both efforts were collected and relayed immediately for display on the Storm-Tide Mapper, which allowed FEMA and other emergency management officials to examine the data and best direct federal response activities. Data collected from this event also permitted the evaluation of the performance of storm-tide models for maximum and incremental water level and flood extent, and the site-specific effects of storm tide on natural and anthropogenic features of the environment.

The locations of water-level and wave-height data collection were selected to augment existing tide-gage networks and to ensure adequate monitoring in areas forecasted to have significant storm tide. After Hurricane Sandy made landfall in New Jersey, sustained winds increased as an effect of an additional storm approaching from the west. The combination of storms, timed with the full-moon high-tide on October 29, exacerbated storm-tide flooding along the New Jersey, New York, and Connecticut coastlines, and caused significant backwater to occur far inland along the Delaware and Hudson Rivers. Storm effects along the Hudson River were measured as far inland as Albany, New York. 


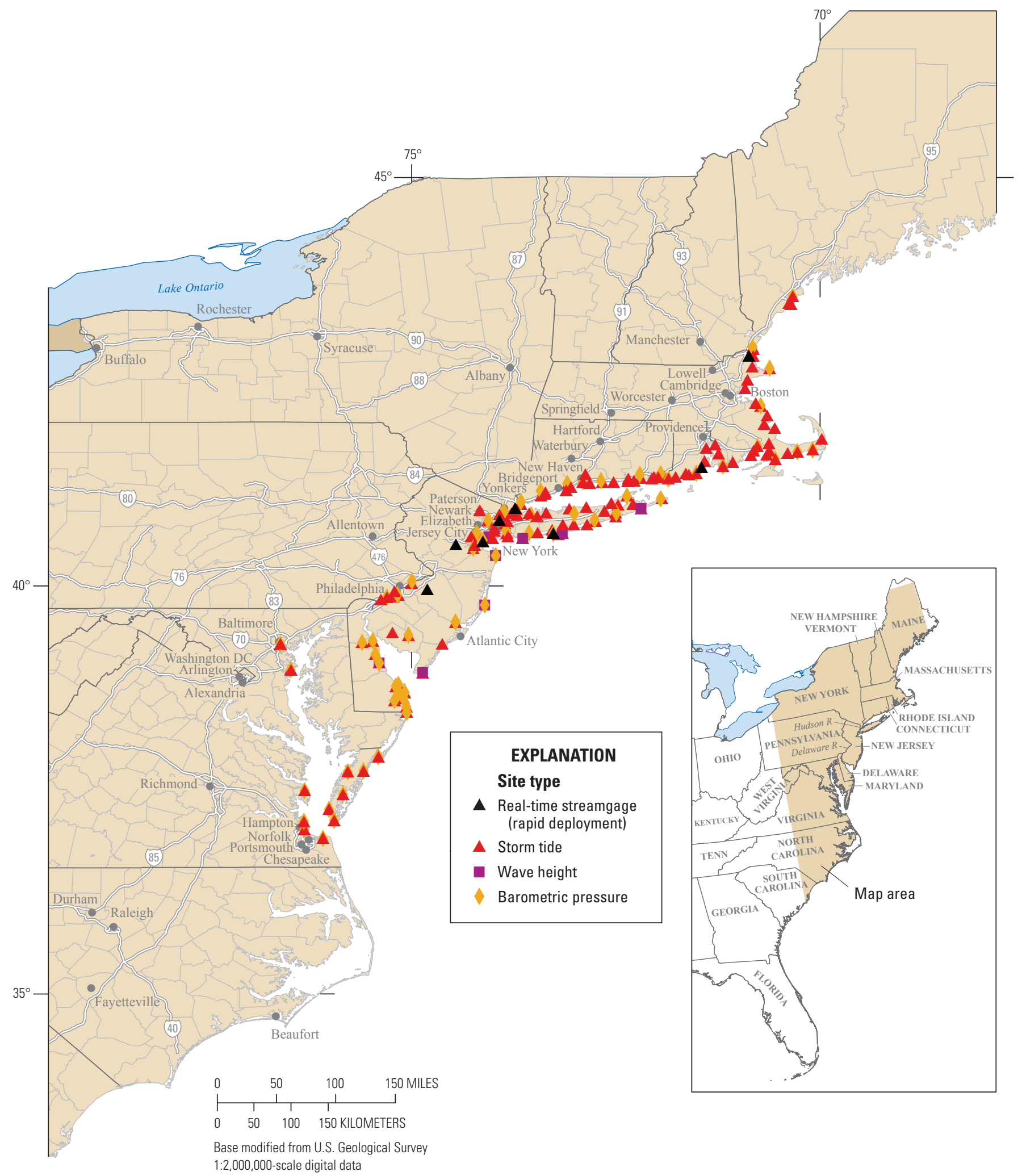

Figure 1. Locations of storm-tide sensors deployed for Hurricane Sandy, October 2012. 
A total of 224 temporarily deployed sensors were used for Hurricane Sandy, 162 water-level and wave-height sensors were deployed at 147 locations (fig. 1). An additional 62 sensors were deployed at additional locations that recorded barometric pressure at 30-second intervals, expressed in pounds per square inch (table 1).

Of the 162 water-level sensors, 145 sensors were programmed to record water pressure at 30 -second intervals, and 9 sensors recorded wave-height measured every 2 seconds, both expressed as water level in feet above the North American Vertical Datum of 1988 (NAVD 88) following surveys completed during sensor recovery. A typical sensor installation is shown in figure 2. An example of water-level elevation and barometric pressure data collected is shown in figure 3. An additional eight locations were rapid-deployment gages (RDG) instrumented with real-time telemetered sensors that recorded water-level elevations and meteorological data every 15 minutes during the hurricane and transmitted hourly to USGS Webpages (table 1; fig. 4).

At the 147 locations, 8 sensors ( 7 water-level and 1 wave-height) were co-located as redundant sensors for quality-control purposes, and an additional 7 wave-height sensors were co-located with storm-tide water-level sensors (162 water-level sensors total). Of the 162 sensors, 7 waterlevel sensors were lost or the structure they were attached to was damaged during the storm, and 5 sensors (4 storm-tide and 1 wave-height) did not record storm tide either due to a lack of water-level rise or because the sensor was installed too high above the water. Two RDGs were destroyed by the flood.

Data were collected and processed following protocols established by McGee and others (2006) and expanded upon by McCallum and others (2012), which included correcting water pressure for changes in barometric pressure and salinity. Quality-control checks were made by (1) deploying redundant sensors at a subset of sites, (2) comparing water levels computed from temporary sensors to water levels recorded at nearby USGS streamgaging and NOAA and USGS tidal stations, and (3) comparing elevations to independent highwater marks where possible. In the aftermath of the storm, 653 independent HWM locations were surveyed relative to NAVD 88 from Virginia to Massachusetts, with particular emphasis in New Jersey and New York where the impacts of the storm were the most pronounced. Multiple HWMs were flagged at some locations. Any HWM was considered an independent location if separated by more than 1,000 feet distance from other HWMs. An additional 297 HWMs were flagged throughout Connecticut, Rhode Island, and Massachusetts but not surveyed due to a lack of funding.
Table 1. Number of sites equipped to monitor Hurricane Sandy storm tide, by State.

\begin{tabular}{lrccc}
\hline & \multicolumn{4}{c}{ Type and number of sensors deployed } \\
\cline { 2 - 5 } \multicolumn{1}{c}{ State } & $\begin{array}{c}\text { Storm } \\
\text { tide }\end{array}$ & $\begin{array}{c}\text { Wave } \\
\text { height }\end{array}$ & $\begin{array}{c}\text { Real-time } \\
\text { rapid } \\
\text { deployment } \\
\text { gages }\end{array}$ & $\begin{array}{c}\text { Barometric } \\
\text { pressure }\end{array}$ \\
\hline Virginia & 10 & 0 & 0 & 10 \\
Maryland & 4 & 0 & 0 & 2 \\
Delaware & 12 & 1 & 0 & 12 \\
Pennsylvania & 6 & 0 & 0 & 3 \\
New Jersey & 10 & 4 & 2 & 7 \\
New York & 39 & 4 & 4 & 11 \\
Connecticut & 27 & 0 & 0 & 7 \\
Rhode Island & 10 & 0 & 1 & 3 \\
Massachusetts & 22 & 0 & 1 & 5 \\
New Hampshire & 2 & 0 & 0 & 1 \\
Maine & 3 & 0 & 0 & 1 \\
Total & 145 & 9 & 8 & 62 \\
\hline
\end{tabular}

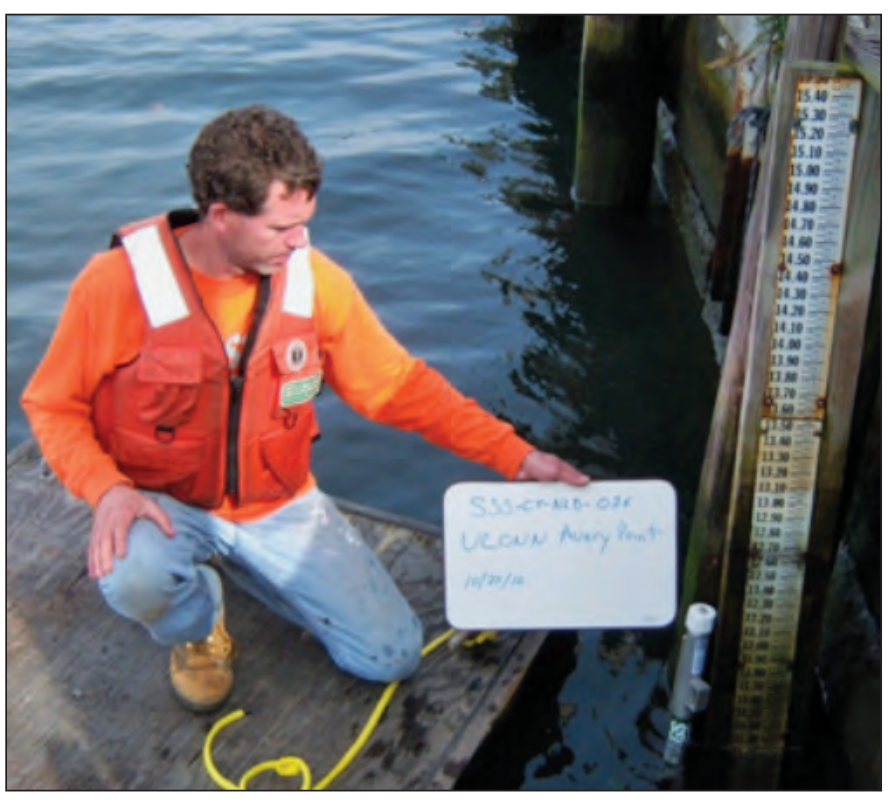

Figure 2. A USGS scientist obtaining water-surface elevation data relative to a known reference elevation and deploying a stormtide sensor at SSS-CT-NLD-025 Poquonock River at Avery Point, Connecticut, October 27, 2012 (photo by Jonathan Morrison, USGS). 


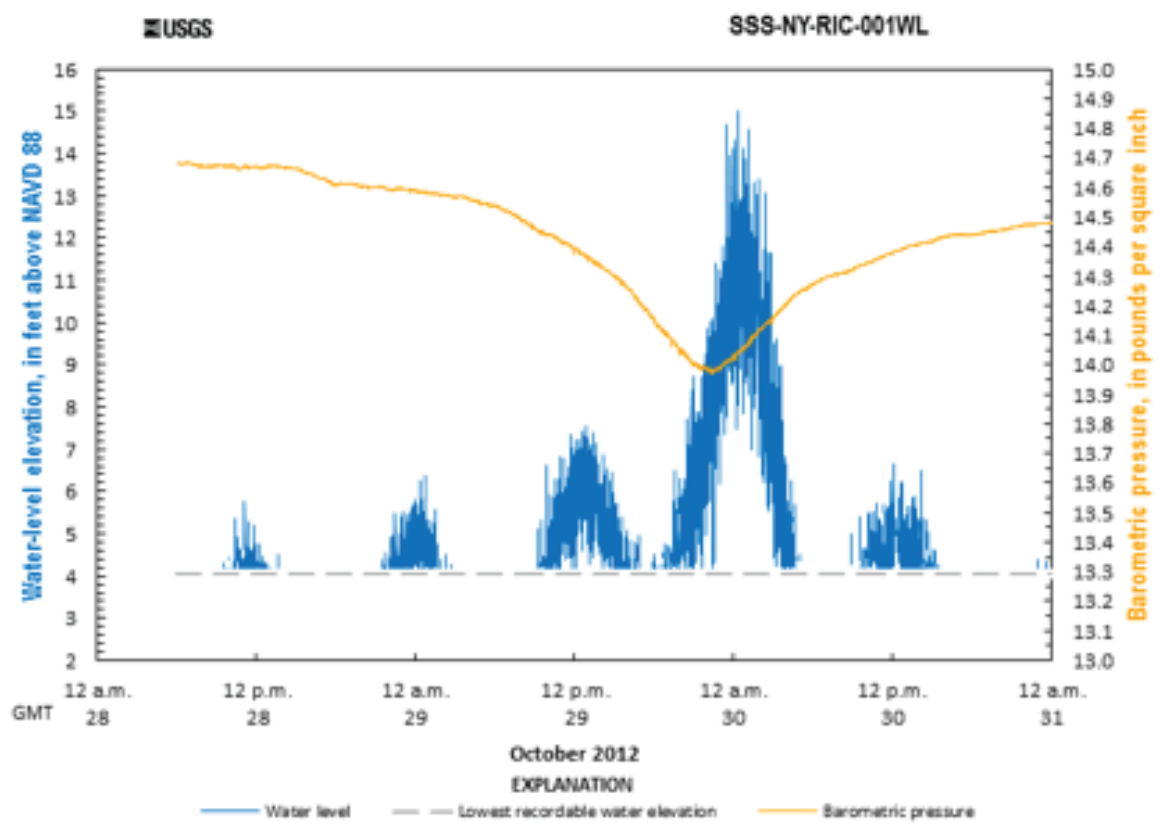

Figure 3. Example of a hydrograph displaying storm-tide elevation and barometric pressure data recorded at SSS-NY-RIC-001WL Lower New York Bay at South Beach at Staten Island, New York during Hurricane Sandy.

Figure 4. A USGS rapiddeployment gage installed to collect real-time water-level and meteorological data at 404810735538063 Harlem River at Randall's Island at Manhattan, New York (photo by Christopher J. Henry, USGS).

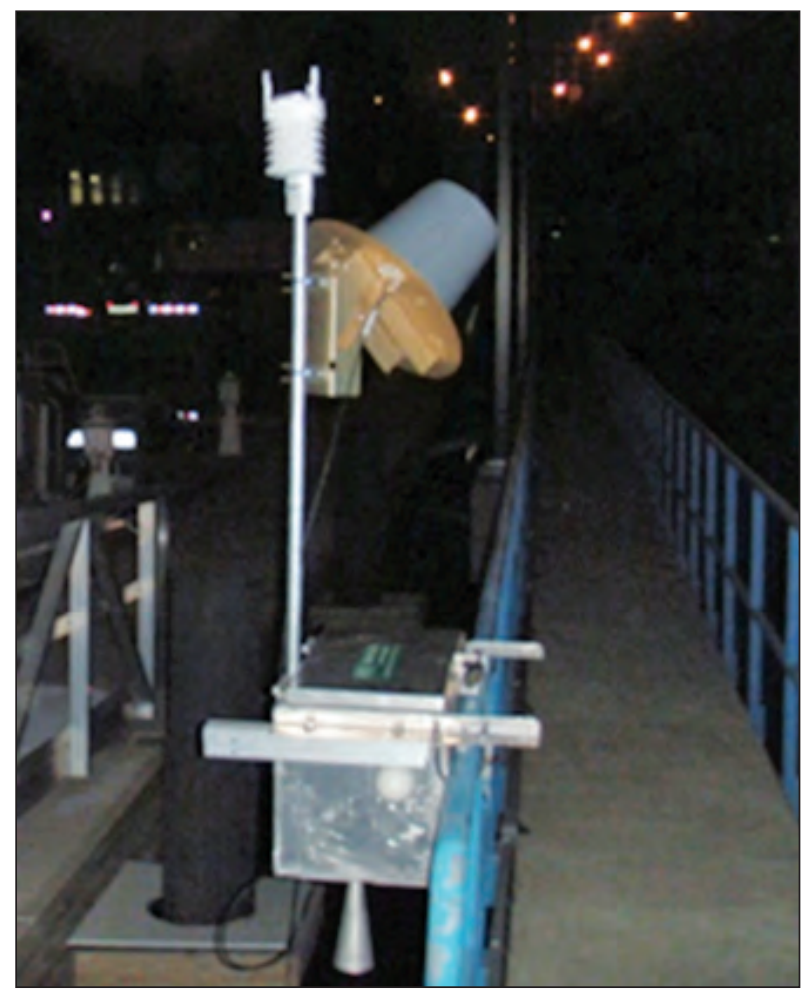




\section{Elevation Surveys}

The water-level data collected by the sensors were initially referenced to points on permanent objects near the water-level sensors in order to establish a temporary datum. After the storm, survey-grade Global Navigation Satellite Systems (GNSS) equipment (fig. 5) was used to determine the elevation above NAVD 88 of the reference points and HWMs, per Rydland and Densmore (2012), and the water-level data were adjusted accordingly to yield elevations relative to NAVD 88 . Some of the water-level sensors for this study were placed in the same locations used during the USGS storm-tide data collection for Hurricane Irene (McCallum and others, 2012), so the water-level sensors were referenced to existing reference points using differential-level surveys or graduated steel tapes. GNSS equipment relies on GEOID models to determine elevations above NAVD 88. The GEOID09 model was used for consistency within the deployment area, and all elevations in this report were derived using the GEOID09 model.

National Geodetic Survey (NGS) benchmarks throughout the study area were surveyed for vertical control. Table 2 lists the GNSS-derived elevations using the GEOID09 model for all NGS benchmarks surveyed during this study and the published NGS elevations from the datasheets, which can be can be accessed at http://www.ngs.noaa.gov/cgi-bin/datasheet.prl. (Tables 2-6 are in the back of the report.)

\section{Data Presentation}

The data from the Hurricane Sandy storm-tide network constitute an extensive collection of continuous water-level records documenting a single, landfall hurricane. The data can be used to evaluate the performance of storm-tide models for maximum and incremental water level and flood extent, and for site-specific effects of storm tide on natural and anthropogenic features of the environment. The data are available in tab-delineated, ASCII format by site for each sensor by using a USGS interactive storm-tide mapper at http://water.usgs.gov/ floods/events/2012/sandy/sandymapper.html.

Digital photographs for selected locations are available on the interactive mapper. Data for each sensor include location, date, time, water level, and barometric pressure, with the data-processing date provided in the file header. Data for each HWM include location, description and quality of the mark, and elevation.

A list of the 162 water-level and wave-height sensor locations and the peak storm tide recorded at each is presented in table 3. Locations were categorized as storm tide, riverine, or wave height as a result of data-collection intervals and

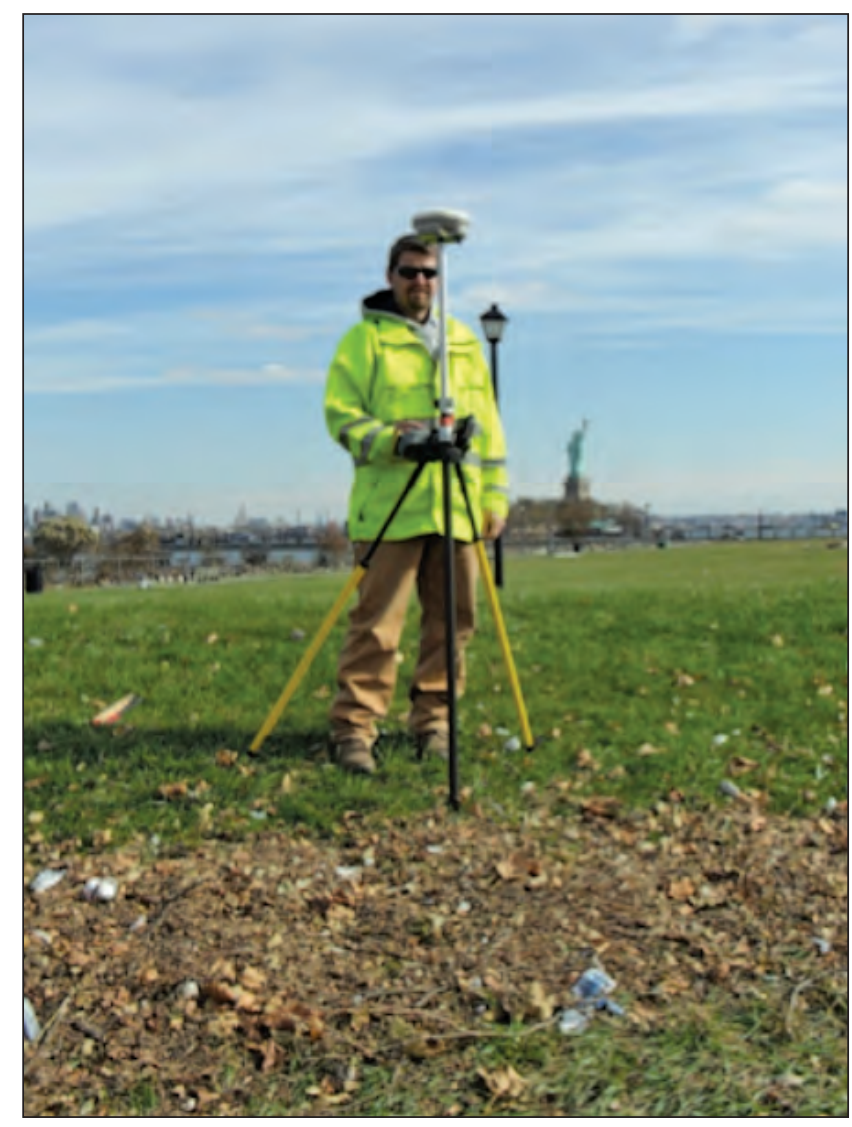

Figure 5. A global positioning system used to survey stormtide elevation at HWM-NJ-HUD-108 at Liberty State Park in Jersey City, New Jersey, after Hurricane Sandy (photo by Crystal Hammer, USGS).

proximity to the ocean. Riverine sites can be influenced by upstream runoff from inland flooding. These temporary sensors were deployed to augment long-term monitoring networks operated by the USGS (table 4) and NOAA (table 5). All HWM data collected by the USGS immediately after Hurricane Sandy are listed in table 6. The reference points and HWMs were surveyed to a vertical accuracy of 0.26 foot at the 95-percent confidence level and within 10 feet horizontally, except for Union, Middlesex, and Monmouth Counties in New Jersey. The vertical accuracy in these counties is 0.47 foot at the 95-percent confidence level. The elevations derived from the GEOID09 model in these three counties are 0.2 to 0.4 foot lower than the published NGS elevations, resulting in a lower accuracy (table 2). The accuracy is computed based on the National Standard for Space Data Accuracy method documented in Federal Geographic Data Committee (1998). 


\section{Acknowledgments}

This report was prepared in cooperation with the Federal Emergency Management Agency. The data in this report are the result of the long hours and extraordinary effort by numerous USGS staff from 15 different States. The authors particularly acknowledge the leadership and dedication of USGS employees in the Wisconsin Water Science Center who assisted with the Storm-Tide Mapper updates to deliver these valuable data to numerous stakeholders in a very timely and accurate manner.

\section{References Cited}

Federal Geographic Data Committee, 1998, Geospatial positioning accuracy standards, part 3-National standard for spatial data accuracy: Federal Geographic Data Comittee, U.S. Geological Survey, 28 p., http://www.fgdc.gov/standards/projects/FGDCstandards-projects/accuracy/part3/chapter3.

McGee, B.D., Goree, B.B., Tollett, R.W., Woodward, B.K., and Kress, W.H., 2006, Hurricane Rita surge data, southwestern Louisiana and southeastern Texas, September to November 2005: U.S. Geological Survey Data Series 220, http://pubs.usgs.gov/ds/2006/220/.
McCallum, B.E., Painter, J.A., and Frantz, E.R., 2012, Monitoring storm tide and flooding from Hurricane Irene along the Atlantic coast of the United States, August 2011: U.S. Geological Survey Open-File Report 2012-1022, http://pubs.usgs.gov/of/2012/1022/.

National Geodetic Survey, 2012, NOTICE: NGS update, September 11, 2012, GEOID12A model released: The NGS Geoid Page, http://www.ngs.noaa.gov/GEOID/.

National Oceanic and Atmospheric Administration, 2008, Tides \& currents: National Oceanic and Atmospheric Administration, accessed October 9, 2012, at http://tidesandcurrents.noaa.gov/index.shtml.

National Weather Service, 1972, The Saffir-Simpson Hurricane Wind Scale [updated February 1, 2012]: National Hurricane Center, accessed October 10, 2012, at http://www.nhc.noaa.gov/aboutsshws.php.

Rydlund, P.H., Jr., and Densmore, B.K., 2012, Methods of practice and guidelines for using survey-grade global navigation satellite systems (GNSS) to establish vertical datum in the United States Geological Survey: U.S. Geological Survey Techniques and Methods, book 11, chap. D1, 102 p. with appendixes, http://pubs.usgs.gov/tm/11d1/tm11-D1.pdf. 
Tables 2-6 
Table 2. Differences between Global Navigation Satellite System (GNSS) surveyed elevations and National Geodetic Survey (NGS) elevations used to check vertical accuracies in New Jersey and New York following Hurricane Sandy, October 2012.

[NGS PID, National Geodetic Survey Point Identifier; elevations referenced to the North American Verical Datum of 1988; GEOID09 is a model]

\begin{tabular}{|c|c|c|c|c|c|}
\hline NGS PID & State & County & $\begin{array}{l}\text { GNSS elevation } \\
\text { (GEOID09), } \\
\text { in feet }\end{array}$ & $\begin{array}{l}\text { NGS datasheet } \\
\text { elevation, } \\
\text { in feet }\end{array}$ & $\begin{array}{c}\text { Difference from } \\
\text { NGS datasheet } \\
\text { (GEOID09), } \\
\text { in feet }\end{array}$ \\
\hline AI8458 & New Jersey & Bergen & 4.72 & 4.90 & -0.18 \\
\hline JU4087 & New Jersey & Burlington & 46.95 & 47.00 & -0.05 \\
\hline JU4087 & New Jersey & Burlington & 46.95 & 47.00 & -0.05 \\
\hline JU4087 & New Jersey & Burlington & 46.97 & 47.00 & -0.03 \\
\hline $\mathrm{AB} 6713$ & New Jersey & Cape May & 10.07 & 10.21 & -0.14 \\
\hline AB6713 & New Jersey & Cape May & 10.17 & 10.21 & -0.04 \\
\hline AJ8715 & New Jersey & Cape May & 32.97 & 32.97 & 0.00 \\
\hline AM6984 & New Jersey & Cape May & 7.23 & 7.29 & -0.06 \\
\hline HU1197 & New Jersey & Cape May & 7.03 & 6.98 & 0.05 \\
\hline KV0282 & New Jersey & Hudson & 36.52 & 36.61 & -0.09 \\
\hline KV0334 & New Jersey & Hudson & 26.79 & 26.92 & -0.13 \\
\hline KV0457 & New Jersey & Hudson & 8.55 & 8.72 & -0.17 \\
\hline KV0457 & New Jersey & Hudson & 8.54 & 8.72 & -0.18 \\
\hline AG9916 & New Jersey & Middlesex & 82.44 & 82.65 & -0.21 \\
\hline KV0015 & New Jersey & Middlesex & 113.80 & 114.05 & -0.25 \\
\hline KV0015 & New Jersey & Middlesex & 113.79 & 114.05 & -0.26 \\
\hline KV0931 & New Jersey & Middlesex & 13.84 & 14.14 & -0.30 \\
\hline DG9900 & New Jersey & Monmouth & 5.58 & 5.74 & -0.16 \\
\hline DG9900 & New Jersey & Monmouth & 5.59 & 5.74 & -0.15 \\
\hline DG9900 & New Jersey & Monmouth & 5.57 & 5.74 & -0.17 \\
\hline DG9900 & New Jersey & Monmouth & 5.55 & 5.74 & -0.19 \\
\hline DM6974 & New Jersey & Monmouth & 11.13 & 11.43 & -0.30 \\
\hline DM6974 & New Jersey & Monmouth & 11.19 & 11.43 & -0.24 \\
\hline DM6981 & New Jersey & Monmouth & 16.06 & 16.38 & -0.32 \\
\hline DM6981 & New Jersey & Monmouth & 16.17 & 16.38 & -0.21 \\
\hline KV0735 & New Jersey & Monmouth & 8.58 & 8.82 & -0.24 \\
\hline KV0735 & New Jersey & Monmouth & 8.54 & 8.82 & -0.28 \\
\hline KV0765 & New Jersey & Monmouth & 21.15 & 21.57 & -0.42 \\
\hline KV3515 & New Jersey & Monmouth & 11.59 & 11.79 & -0.20 \\
\hline KV3515 & New Jersey & Monmouth & 11.60 & 11.79 & -0.19 \\
\hline KV3515 & New Jersey & Monmouth & 11.65 & 11.79 & -0.14 \\
\hline DM6002 & New Jersey & Ocean & 5.58 & 5.64 & -0.06 \\
\hline JU0067 & New Jersey & Ocean & 17.88 & 17.86 & 0.02 \\
\hline
\end{tabular}


Table 2. Differences between Global Navigation Satellite System (GNSS) surveyed elevations and National Geodetic Survey (NGS) elevations used to check vertical accuracies in New Jersey and New York following Hurricane Sandy, October 2012.-Continued

[NGS PID, National Geodetic Survey Point Identifier; elevations referenced to the North American Verical Datum of 1988; GEOID09 is a model]

\begin{tabular}{|c|c|c|c|c|c|}
\hline NGS PID & State & County & $\begin{array}{l}\text { GNSS elevation } \\
\text { (GEOID09), } \\
\text { in feet }\end{array}$ & $\begin{array}{l}\text { NGS datasheet } \\
\text { elevation, } \\
\text { in feet }\end{array}$ & $\begin{array}{c}\text { Difference from } \\
\text { NGS datasheet } \\
\text { (GEOID09), } \\
\text { in feet }\end{array}$ \\
\hline JU0136 & New Jersey & Ocean & 16.52 & 16.55 & -0.03 \\
\hline KV0838 & New Jersey & Ocean & 36.47 & 36.55 & -0.08 \\
\hline AA5229 & New Jersey & Union & 14.07 & 14.34 & -0.27 \\
\hline KV0061 & New Jersey & Union & 30.08 & 30.28 & -0.20 \\
\hline AB6708 & New Jersey & Atlantic & 64.26 & 64.28 & -0.02 \\
\hline JU0269 & New Jersey & Atlantic & 38.16 & 38.43 & -0.27 \\
\hline JU2456 & New Jersey & Atlantic & 8.76 & 8.90 & -0.14 \\
\hline KU1162 & New York & Bronx & 21.03 & 21.19 & -0.16 \\
\hline KU1162 & New York & Bronx & 21.07 & 21.19 & -0.12 \\
\hline KU1162 & New York & Bronx & 21.03 & 21.19 & -0.16 \\
\hline MZ0734 & New York & Greene & 574.97 & 574.86 & 0.11 \\
\hline MZ3054 & New York & Greene & 25.17 & 25.20 & -0.03 \\
\hline KV0584 & New York & New York & 11.21 & 11.26 & -0.05 \\
\hline KV0584 & New York & New York & 11.23 & 11.26 & -0.03 \\
\hline KU0978 & New York & Queens & 11.16 & 10.84 & 0.32 \\
\hline KU0978 & New York & Queens & 11.06 & 10.84 & 0.22 \\
\hline KU1199 & New York & Queens & 22.63 & 22.85 & -0.22 \\
\hline KU1199 & New York & Queens & 22.75 & 22.85 & -0.10 \\
\hline KU1199 & New York & Queens & 22.59 & 22.85 & -0.26 \\
\hline KU1199 & New York & Queens & 22.69 & 22.85 & -0.16 \\
\hline KU1199 & New York & Queens & 22.67 & 22.85 & -0.18 \\
\hline KU1199 & New York & Queens & 22.72 & 22.85 & -0.13 \\
\hline KU1199 & New York & Queens & 22.71 & 22.85 & -0.14 \\
\hline KU1199 & New York & Queens & 22.50 & 22.85 & -0.35 \\
\hline KU1199 & New York & Queens & 22.77 & 22.85 & -0.08 \\
\hline KU1199 & New York & Queens & 22.86 & 22.85 & 0.01 \\
\hline KU1199 & New York & Queens & 22.61 & 22.85 & -0.24 \\
\hline KU1199 & New York & Queens & 22.73 & 22.85 & -0.12 \\
\hline KU1199 & New York & Queens & 22.91 & 22.85 & 0.06 \\
\hline KU1199 & New York & Queens & 22.96 & 22.85 & 0.11 \\
\hline KU1218 & New York & Queens & 14.70 & 14.89 & -0.19 \\
\hline KU1218 & New York & Queens & 14.75 & 14.89 & -0.14 \\
\hline KU1218 & New York & Queens & 14.76 & 14.89 & -0.13 \\
\hline
\end{tabular}


Table 2. Differences between Global Navigation Satellite System (GNSS) surveyed elevations and National Geodetic Survey (NGS) elevations used to check vertical accuracies in New Jersey and New York following Hurricane Sandy, October 2012.-Continued

[NGS PID, National Geodetic Survey Point Identifier; elevations referenced to the North American Verical Datum of 1988; GEOID09 is a model]

\begin{tabular}{|c|c|c|c|c|c|}
\hline NGS PID & State & County & $\begin{array}{l}\text { GNSS elevation } \\
\text { (GEOID09), } \\
\text { in feet }\end{array}$ & $\begin{array}{l}\text { NGS datasheet } \\
\text { elevation, } \\
\text { in feet }\end{array}$ & $\begin{array}{c}\text { Difference from } \\
\text { NGS datasheet } \\
\text { (GEOID09), } \\
\text { in feet }\end{array}$ \\
\hline KU1218 & New York & Queens & 14.82 & 14.89 & -0.07 \\
\hline KU1218 & New York & Queens & 14.77 & 14.89 & -0.12 \\
\hline KU1218 & New York & Queens & 14.79 & 14.89 & -0.10 \\
\hline KU1218 & New York & Queens & 14.78 & 14.89 & -0.11 \\
\hline KU1218 & New York & Queens & 14.83 & 14.89 & -0.06 \\
\hline KU1218 & New York & Queens & 14.79 & 14.89 & -0.10 \\
\hline KU1218 & New York & Queens & 14.96 & 14.89 & 0.07 \\
\hline KU1218 & New York & Queens & 14.92 & 14.89 & 0.03 \\
\hline KU1218 & New York & Queens & 14.89 & 14.89 & -0.00 \\
\hline KU1218 & New York & Queens & 14.91 & 14.89 & 0.02 \\
\hline KU1218 & New York & Queens & 14.90 & 14.89 & 0.01 \\
\hline KU1218 & New York & Queens & 15.01 & 14.89 & 0.12 \\
\hline LY2033 & New York & Ulster & 353.20 & 353.21 & -0.01 \\
\hline LY2033 & New York & Ulster & 353.18 & 353.21 & -0.03 \\
\hline NA0463 & New York & Ulster & 302.13 & 302.17 & -0.04 \\
\hline KU0953 & New York & Westchester & 7.96 & 8.14 & -0.18 \\
\hline KU0953 & New York & Westchester & 8.02 & 8.14 & -0.12 \\
\hline KU1740 & New York & Westchester & 7.24 & 7.60 & -0.36 \\
\hline MZ1289 & New York & Albany & 38.23 & 38.28 & -0.05 \\
\hline MZ1289 & New York & Albany & 38.16 & 38.28 & -0.12 \\
\hline KU0028 & New York & Suffolk & 39.48 & 39.39 & 0.09 \\
\hline KU0194 & New York & Suffolk & 3.72 & 3.79 & -0.07 \\
\hline KU0743 & New York & Suffolk & 93.14 & 93.05 & 0.09 \\
\hline KU0743 & New York & Suffolk & 93.14 & 93.05 & 0.09 \\
\hline KU0743 & New York & Suffolk & 93.17 & 93.05 & 0.12 \\
\hline KU0743 & New York & Suffolk & 93.13 & 93.05 & 0.08 \\
\hline KU0743 & New York & Suffolk & 92.86 & 93.05 & -0.19 \\
\hline KU0743 & New York & Suffolk & 93.17 & 93.05 & 0.12 \\
\hline KU0743 & New York & Suffolk & 92.95 & 93.05 & -0.10 \\
\hline KU0743 & New York & Suffolk & 93.08 & 93.05 & 0.03 \\
\hline KU0743 & New York & Suffolk & 92.98 & 93.05 & -0.07 \\
\hline
\end{tabular}


Table 3. Hurricane Sandy peak storm-tide data for 165 U.S. Geological Survey temporarily deployed sites, by State.

[NAVD 88, North American Vertical Datum of 1988; GMT, Greenwich Mean Time; RDG, rapid-deployment gage]

\begin{tabular}{|c|c|c|c|c|c|c|c|c|}
\hline $\begin{array}{c}\text { Site } \\
\text { identification }\end{array}$ & State & County & $\begin{array}{l}\text { Latitude } \\
\text { Decima }\end{array}$ & Longitude & Site type & $\begin{array}{c}\text { Type } \\
\text { of data } \\
\text { recorded }\end{array}$ & $\begin{array}{c}\text { Peak } \\
\text { storm-tide } \\
\text { elevation, } \\
\text { feet above } \\
\text { NAVD } 88\end{array}$ & $\begin{array}{c}\text { Peak } \\
\text { storm-tide } \\
\text { date and time } \\
\text { (GMT) }\end{array}$ \\
\hline $\begin{array}{l}\text { SSS-CT-FFD- } \\
001 \mathrm{WL}\end{array}$ & Connecticut & Fairfield & 40.99910 & -73.65944 & water level & storm tide & 10.27 & $\begin{array}{c}10 / 30 / 2012 \\
01: 55: 30\end{array}$ \\
\hline $\begin{array}{l}\text { SSS-CT-FFD- } \\
005 \mathrm{WL}\end{array}$ & Connecticut & Fairfield & 41.14762 & -73.36481 & water level & storm tide & 10.31 & $\begin{array}{c}\text { 10/30/2012 } \\
\text { 02:09:00 }\end{array}$ \\
\hline $\begin{array}{l}\text { SSS-CT-FFD- } \\
\text { 006WL }\end{array}$ & Connecticut & Fairfield & 41.12310 & -73.36998 & water level & storm tide & 10.15 & $\begin{array}{c}10 / 30 / 2012 \\
02: 22: 30\end{array}$ \\
\hline $\begin{array}{l}\text { SSS-CT-FFD- } \\
\text { 009WL }\end{array}$ & Connecticut & Fairfield & 41.25325 & -73.08843 & water level & storm tide & 9.65 & $\begin{array}{c}\text { 10/30/2012 } \\
02: 08: 30\end{array}$ \\
\hline $\begin{array}{l}\text { SSS-CT-FFD- } \\
\text { 012WL }\end{array}$ & Connecticut & Fairfield & 41.16316 & -73.10901 & water level & storm tide & none & $\begin{array}{l}\text { peak not } \\
\text { recorded }\end{array}$ \\
\hline $\begin{array}{l}\text { SSS-CT-MSX- } \\
\text { 018WL }\end{array}$ & Connecticut & Middlesex & 41.26919 & -72.52936 & water level & storm tide & 7.63 & $\begin{array}{c}10 / 30 / 2012 \\
01: 26: 30\end{array}$ \\
\hline $\begin{array}{l}\text { SSS-CT-MSX- } \\
\text { 019WL }\end{array}$ & Connecticut & Middlesex & 41.28111 & -72.35222 & water level & storm tide & 7.73 & $\begin{array}{c}\text { 10/30/2012 } \\
00: 48: 30\end{array}$ \\
\hline $\begin{array}{l}\text { SSS-CT-MSX- } \\
\text { 020WL }\end{array}$ & Connecticut & Middlesex & 41.28111 & -72.35222 & water level & storm tide & 7.86 & $\begin{array}{c}10 / 30 / 2012 \\
01: 24: 50\end{array}$ \\
\hline $\begin{array}{l}\text { SSS-CT-NHV- } \\
\text { 013WL }\end{array}$ & Connecticut & New Haven & 41.27221 & -72.90479 & water level & storm tide & 9.50 & $\begin{array}{c}10 / 30 / 2012 \\
01: 29: 00\end{array}$ \\
\hline $\begin{array}{l}\text { SSS-CT-NHV- } \\
\text { 019WL }\end{array}$ & Connecticut & New Haven & 41.27221 & -72.90479 & water level & storm tide & 9.23 & $\begin{array}{c}\text { 10/30/2012 } \\
01: 29: 00\end{array}$ \\
\hline $\begin{array}{l}\text { SSS-CT-NHV- } \\
\text { 020WL }\end{array}$ & Connecticut & New Haven & 41.21129 & -73.04948 & water level & storm tide & 9.84 & $\begin{array}{c}10 / 30 / 2012 \\
01: 41: 00\end{array}$ \\
\hline $\begin{array}{l}\text { SSS-CT-NLD- } \\
\text { 015WL }\end{array}$ & Connecticut & New London & 41.32525 & -71.98460 & water level & storm tide & 6.35 & $\begin{array}{c}\text { 10/30/2012 } \\
00: 28: 30\end{array}$ \\
\hline $\begin{array}{l}\text { SSS-CT-NLD- } \\
\text { 016WL }\end{array}$ & Connecticut & New London & 41.32525 & -71.98460 & water level & storm tide & 6.42 & $\begin{array}{c}10 / 29 / 2012 \\
22: 46: 40\end{array}$ \\
\hline $\begin{array}{l}\text { SSS-CT-NLD- } \\
\text { 018WL }\end{array}$ & Connecticut & New London & 41.32147 & -72.19540 & water level & storm tide & 11.72 & $\begin{array}{c}10 / 29 / 2012 \\
23: 56: 00\end{array}$ \\
\hline $\begin{array}{l}\text { SSS-CT-NLD- } \\
\text { 019WL }\end{array}$ & Connecticut & New London & 41.28428 & -72.27758 & water level & storm tide & 8.26 & $\begin{array}{c}10 / 30 / 2012 \\
00: 35: 50\end{array}$ \\
\hline $\begin{array}{l}\text { SSS-CT-NLD- } \\
\text { 022WL }\end{array}$ & Connecticut & New London & 41.31250 & -72.34608 & water level & storm tide & 7.01 & $\begin{array}{c}\text { 10/30/2012 } \\
01: 34: 00\end{array}$ \\
\hline $\begin{array}{l}\text { SSS-CT-NLD- } \\
\text { 023WL }\end{array}$ & Connecticut & New London & 41.28428 & -72.27758 & water level & storm tide & 8.41 & $\begin{array}{c}\text { 10/30/2012 } \\
\text { 00:09:30 }\end{array}$ \\
\hline
\end{tabular}


Table 3. Hurricane Sandy peak storm-tide data for 165 U.S. Geological Survey temporarily deployed sites, by State.—Continued [NAVD 88, North American Vertical Datum of 1988; GMT, Greenwich Mean Time; RDG, rapid-deployment gage]

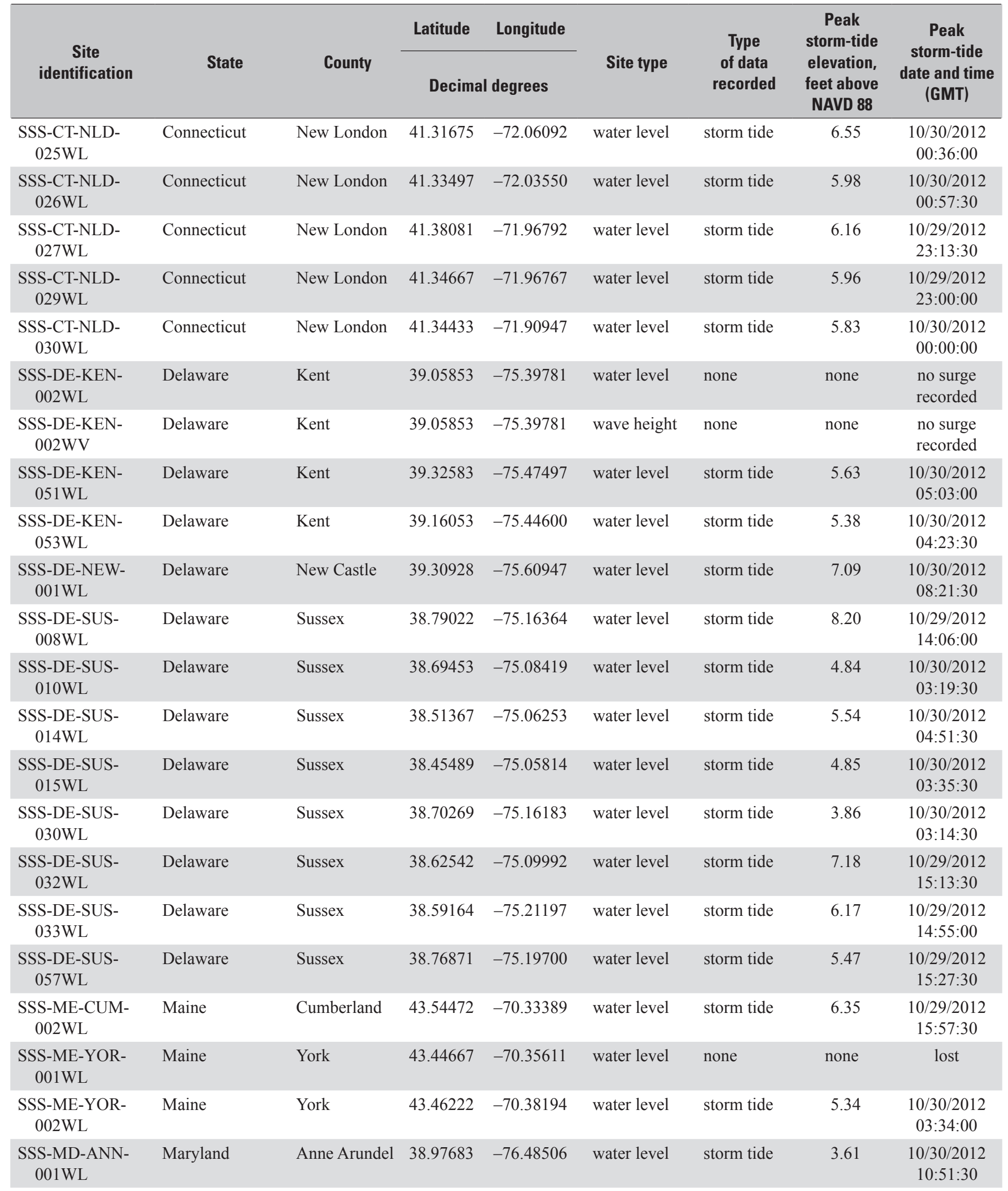


Table 3. Hurricane Sandy peak storm-tide data for 165 U.S. Geological Survey temporarily deployed sites, by State.—Continued [NAVD 88, North American Vertical Datum of 1988; GMT, Greenwich Mean Time; RDG, rapid-deployment gage]

\begin{tabular}{|c|c|c|c|c|c|c|c|c|}
\hline $\begin{array}{c}\text { Site } \\
\text { identification }\end{array}$ & State & County & $\begin{array}{c}\text { Latitude } \\
\text { Decima }\end{array}$ & Longitude & Site type & $\begin{array}{c}\text { Type } \\
\text { of data } \\
\text { recorded }\end{array}$ & $\begin{array}{c}\text { Peak } \\
\text { storm-tide } \\
\text { elevation, } \\
\text { feet above } \\
\text { NAVD } 88\end{array}$ & $\begin{array}{c}\text { Peak } \\
\text { storm-tide } \\
\text { date and time } \\
\text { (GMT) }\end{array}$ \\
\hline $\begin{array}{l}\text { SSS-MD-ANN- } \\
003 \mathrm{WL}\end{array}$ & Maryland & Anne Arundel & 38.96861 & -76.47631 & water level & storm tide & 3.41 & $\begin{array}{c}10 / 30 / 2012 \\
10: 46: 00\end{array}$ \\
\hline $\begin{array}{l}\text { SSS-MD-BAL- } \\
\text { 003WL }\end{array}$ & Maryland & Baltimore & 39.28636 & -76.60581 & water level & none & none & $\begin{array}{l}\text { no surge } \\
\text { recorded }\end{array}$ \\
\hline 424858070522116 & Massachusetts & Essex & 42.81612 & -70.87255 & $\begin{array}{l}\text { real-time } \\
\text { RDG }\end{array}$ & storm tide & 6.68 & $\begin{array}{c}10 / 29 / 2012 \\
16: 15: 00\end{array}$ \\
\hline $\begin{array}{l}\text { SSS-MA-BAR- } \\
023 \mathrm{WL}\end{array}$ & Massachusetts & Barnstable & 41.65638 & -70.62355 & water level & storm tide & 5.29 & $\begin{array}{c}10 / 29 / 2012 \\
23: 08: 30\end{array}$ \\
\hline $\begin{array}{l}\text { SSS-MA-BAR- } \\
\text { 025WL }\end{array}$ & Massachusetts & Barnstable & 41.55283 & -70.54864 & water level & storm tide & 3.59 & $\begin{array}{c}\text { 10/29/2012 } \\
22: 00: 00\end{array}$ \\
\hline $\begin{array}{l}\text { SSS-MA-BAR- } \\
\text { 026WL }\end{array}$ & Massachusetts & Barnstable & 41.62131 & -70.39557 & water level & storm tide & 3.57 & $\begin{array}{c}10 / 29 / 2012 \\
17: 05: 30\end{array}$ \\
\hline $\begin{array}{l}\text { SSS-MA-BAR- } \\
027 \mathrm{WL}\end{array}$ & Massachusetts & Barnstable & 41.66752 & -70.08976 & water level & storm tide & 3.45 & $\begin{array}{c}\text { 10/29/2012 } \\
17: 00: 00\end{array}$ \\
\hline $\begin{array}{l}\text { SSS-MA-BAR- } \\
028 \mathrm{WL}\end{array}$ & Massachusetts & Barnstable & 41.63949 & -70.27664 & water level & storm tide & 3.95 & $\begin{array}{c}10 / 29 / 2012 \\
17: 33: 00\end{array}$ \\
\hline $\begin{array}{l}\text { SSS-MA-BAR- } \\
\text { 031WL }\end{array}$ & Massachusetts & Barnstable & 41.80028 & -69.98068 & water level & storm tide & 7.06 & $\begin{array}{c}10 / 29 / 2012 \\
16: 53: 30\end{array}$ \\
\hline $\begin{array}{l}\text { SSS-MA-ESS- } \\
\text { 038WL }\end{array}$ & Massachusetts & Essex & 42.51951 & -70.88681 & water level & storm tide & 6.91 & $\begin{array}{c}10 / 29 / 2012 \\
15: 22: 30\end{array}$ \\
\hline $\begin{array}{l}\text { SSS-MA-ESS- } \\
\text { 039WL }\end{array}$ & Massachusetts & Essex & 42.65899 & -70.61502 & water level & storm tide & 6.83 & $\begin{array}{c}10 / 29 / 2012 \\
16: 06: 00\end{array}$ \\
\hline $\begin{array}{l}\text { SSS-MA-ESS- } \\
\text { 040WL }\end{array}$ & Massachusetts & Essex & 42.68369 & -70.82777 & water level & storm tide & 7.12 & $\begin{array}{c}10 / 29 / 2012 \\
16: 11: 00\end{array}$ \\
\hline $\begin{array}{l}\text { SSS-MA-ESS- } \\
\text { 041WL }\end{array}$ & Massachusetts & Essex & 42.81693 & -70.82020 & water level & storm tide & 7.35 & $\begin{array}{c}10 / 29 / 2012 \\
16: 16: 30\end{array}$ \\
\hline $\begin{array}{l}\text { SSS-MA-NOR- } \\
\text { 036WL }\end{array}$ & Massachusetts & Norfolk & 42.23905 & -70.78930 & water level & storm tide & 6.89 & $\begin{array}{c}10 / 29 / 2012 \\
15: 53: 30\end{array}$ \\
\hline $\begin{array}{l}\text { SSS-MA-PLY- } \\
\text { 018WL }\end{array}$ & Massachusetts & Plymouth & 41.65608 & -70.81283 & water level & storm tide & 6.90 & $\begin{array}{c}10 / 29 / 2012 \\
23: 46: 30\end{array}$ \\
\hline $\begin{array}{l}\text { SSS-MA-PLY- } \\
\text { 019WL }\end{array}$ & Massachusetts & Plymouth & 41.71257 & -70.76535 & water level & storm tide & 5.56 & $\begin{array}{c}10 / 29 / 2012 \\
23: 26: 30\end{array}$ \\
\hline $\begin{array}{l}\text { SSS-MA-PLY- } \\
\text { 021WL }\end{array}$ & Massachusetts & Plymouth & 41.74369 & -70.62779 & water level & storm tide & 4.91 & $\begin{array}{c}10 / 29 / 2012 \\
23: 52: 30\end{array}$ \\
\hline
\end{tabular}


Table 3. Hurricane Sandy peak storm-tide data for 165 U.S. Geological Survey temporarily deployed sites, by State.—Continued [NAVD 88, North American Vertical Datum of 1988; GMT, Greenwich Mean Time; RDG, rapid-deployment gage]

\begin{tabular}{|c|c|c|c|c|c|c|c|c|}
\hline $\begin{array}{c}\text { Site } \\
\text { identification }\end{array}$ & State & County & $\begin{array}{c}\text { Latitude } \\
\text { Decima }\end{array}$ & Longitude & Site type & $\begin{array}{c}\text { Type } \\
\text { of data } \\
\text { recorded }\end{array}$ & $\begin{array}{c}\text { Peak } \\
\text { storm-tide } \\
\text { elevation, } \\
\text { feet above } \\
\text { NAVD } 88\end{array}$ & $\begin{array}{c}\text { Peak } \\
\text { storm-tide } \\
\text { date and time } \\
\text { (GMT) }\end{array}$ \\
\hline $\begin{array}{l}\text { SSS-MA-PLY- } \\
\text { 032WL }\end{array}$ & Massachusetts & Plymouth & 41.92901 & -70.55507 & water level & storm tide & 8.05 & $\begin{array}{c}10 / 29 / 2012 \\
15: 31: 00\end{array}$ \\
\hline $\begin{array}{l}\text { SSS-MA-PLY- } \\
\text { 033WL }\end{array}$ & Massachusetts & Plymouth & 41.98024 & -70.68291 & water level & storm tide & 8.31 & $\begin{array}{c}10 / 29 / 2012 \\
15: 35: 00\end{array}$ \\
\hline $\begin{array}{l}\text { SSS-MA-PLY- } \\
\text { 034WL }\end{array}$ & Massachusetts & Plymouth & 42.08254 & -70.64636 & water level & storm tide & 7.30 & $\begin{array}{c}10 / 29 / 2012 \\
15: 51: 00\end{array}$ \\
\hline $\begin{array}{l}\text { SSS-MA-PLY- } \\
\text { 035WL }\end{array}$ & Massachusetts & Plymouth & 42.20139 & -70.72573 & water level & storm tide & 7.86 & $\begin{array}{c}10 / 29 / 2012 \\
15: 34: 00\end{array}$ \\
\hline $\begin{array}{l}\text { SSS-NH-ROC- } \\
\text { 004WL }\end{array}$ & New Hampshire & Rockingham & 42.89820 & -70.81700 & water level & storm tide & 7.19 & $\begin{array}{c}10 / 29 / 2012 \\
19: 49: 00\end{array}$ \\
\hline $\begin{array}{l}\text { SSS-NH-ROC- } \\
\text { 005WL }\end{array}$ & New Hampshire & Rockingham & 42.92180 & -70.82330 & water level & storm tide & 6.04 & $\begin{array}{c}10 / 29 / 2012 \\
20: 26: 00\end{array}$ \\
\hline 395740074482628 & New Jersey & Burlington & 39.96137 & -74.80724 & $\begin{array}{l}\text { real-time } \\
\text { RDG }\end{array}$ & storm tide & 7.14 & $\begin{array}{c}10 / 30 / 2012 \\
12: 15: 00\end{array}$ \\
\hline 403030074273017 & New Jersey & Middlesex & 40.50843 & -74.45838 & $\begin{array}{l}\text { real-time } \\
\text { RDG }\end{array}$ & storm tide & 13.88 & $\begin{array}{c}10 / 30 / 2012 \\
02: 15: 00\end{array}$ \\
\hline $\begin{array}{l}\text { SSS-NJ-ATL- } \\
005 \mathrm{WL}\end{array}$ & New Jersey & Atlantic & 39.55333 & -74.46278 & water level & storm tide & 7.92 & $\begin{array}{c}10 / 30 / 2012 \\
02: 06: 30\end{array}$ \\
\hline $\begin{array}{l}\text { SSS-NJ-CPM- } \\
\text { 010WL }\end{array}$ & New Jersey & Cape May & 39.28833 & -74.62750 & water level & storm tide & 6.97 & $\begin{array}{c}10 / 30 / 2012 \\
00: 42: 00\end{array}$ \\
\hline $\begin{array}{l}\text { SSS-NJ-CPM- } \\
\text { 035WL }\end{array}$ & New Jersey & Cape May & 38.93639 & -74.86556 & water level & storm tide & 12.89 & $\begin{array}{c}10 / 29 / 2012 \\
13: 28: 30\end{array}$ \\
\hline $\begin{array}{l}\text { SSS-NJ-CPM- } \\
035 \mathrm{WV}\end{array}$ & New Jersey & Cape May & 38.93639 & -74.86556 & wave height & $\begin{array}{l}\text { wave } \\
\text { height }\end{array}$ & 12.55 & $\begin{array}{c}10 / 29 / 2012 \\
14: 05: 52\end{array}$ \\
\hline $\begin{array}{l}\text { SSS-NJ-CUM- } \\
\text { 020WL }\end{array}$ & New Jersey & Cumberland & 39.39528 & -75.04083 & water level & storm tide & 6.31 & $\begin{array}{c}\text { 10/30/2012 } \\
\text { 06:08:00 }\end{array}$ \\
\hline $\begin{array}{l}\text { SSS-NJ-CUM- } \\
025 \mathrm{WL}\end{array}$ & New Jersey & Cumberland & 39.42917 & -75.23694 & water level & storm tide & 6.44 & $\begin{array}{c}\text { 10/30/2012 } \\
06: 59: 00\end{array}$ \\
\hline $\begin{array}{l}\text { SSS-NJ-HUD- } \\
002 \mathrm{WL}\end{array}$ & New Jersey & Hudson & 40.79982 & -74.06606 & water level & storm tide & 8.80 & $\begin{array}{c}\text { 10/30/2012 } \\
\text { 03:09:00 }\end{array}$ \\
\hline $\begin{array}{l}\text { SSS-NJ-MID- } \\
\text { 001WL }\end{array}$ & New Jersey & Middlesex & 40.45911 & -74.24687 & water level & storm tide & 11.71 & $\begin{array}{c}10 / 29 / 2012 \\
23: 02: 30\end{array}$ \\
\hline $\begin{array}{l}\text { SSS-NJ-MON- } \\
002 \mathrm{WV}\end{array}$ & New Jersey & Monmouth & 40.37222 & -73.97304 & wave height & $\begin{array}{l}\text { wave } \\
\text { height }\end{array}$ & 17.05 & $\begin{array}{c}10 / 29 / 2012 \\
23: 30: 16\end{array}$ \\
\hline $\begin{array}{l}\text { SSS-NJ-MON- } \\
003 \mathrm{WV}\end{array}$ & New Jersey & Monmouth & 40.37222 & -73.97304 & wave height & $\begin{array}{l}\text { wave } \\
\text { height }\end{array}$ & 19.50 & $\begin{array}{c}10 / 30 / 2012 \\
00: 40: 56\end{array}$ \\
\hline $\begin{array}{l}\text { SSS-NJ-OCE- } \\
001 \mathrm{WV}\end{array}$ & New Jersey & Ocean & 39.76361 & -74.10417 & wave height & $\begin{array}{l}\text { wave } \\
\text { height }\end{array}$ & 8.96 & $\begin{array}{c}10 / 30 / 2012 \\
00: 44: 02\end{array}$ \\
\hline $\begin{array}{l}\text { SSS-NJ-PAS- } \\
001 \mathrm{WL}\end{array}$ & New Jersey & Passaic & 40.92647 & 74.16631 & water level & riverine & none & $\begin{array}{l}\text { no surge } \\
\text { recorded }\end{array}$ \\
\hline $\begin{array}{l}\text { SSS-NJ-UNI- } \\
001 \mathrm{WL}\end{array}$ & New Jersey & Union & 40.64775 & -74.20512 & water level & storm tide & 12.20 & $\begin{array}{c}10 / 30 / 2012 \\
01: 37: 30\end{array}$ \\
\hline $\begin{array}{l}\text { SSS-NJ-UNI- } \\
002 \mathrm{WL}\end{array}$ & New Jersey & Union & 40.59952 & -74.27177 & water level & storm tide & 12.60 & $\begin{array}{c}10 / 30 / 2012 \\
02: 21: 00\end{array}$ \\
\hline
\end{tabular}


Table 3. Hurricane Sandy peak storm-tide data for 165 U.S. Geological Survey temporarily deployed sites, by State.—Continued [NAVD 88, North American Vertical Datum of 1988; GMT, Greenwich Mean Time; RDG, rapid-deployment gage]

\begin{tabular}{|c|c|c|c|c|c|c|c|c|}
\hline $\begin{array}{c}\text { Site } \\
\text { identification }\end{array}$ & State & County & \multicolumn{2}{|c|}{ Decimal degrees } & Site type & $\begin{array}{c}\text { Type } \\
\text { of data } \\
\text { recorded }\end{array}$ & $\begin{array}{c}\text { Peak } \\
\text { storm-tide } \\
\text { elevation, } \\
\text { feet above } \\
\text { NAVD } 88\end{array}$ & $\begin{array}{c}\text { Peak } \\
\text { storm-tide } \\
\text { date and time } \\
\text { (GMT) }\end{array}$ \\
\hline 403236074073983 & New York & Richmond & 40.54340 & -74.12773 & $\begin{array}{l}\text { real-time } \\
\text { RDG }\end{array}$ & storm tide & none & $\begin{array}{l}\text { peak not } \\
\text { recorded }\end{array}$ \\
\hline 404810735538063 & New York & New York & 40.80059 & -73.92647 & $\begin{array}{l}\text { real-time } \\
\text { RDG }\end{array}$ & storm tide & none & $\begin{array}{l}\text { peak not } \\
\text { recorded }\end{array}$ \\
\hline 405658073433147 & New York & Westchester & 40.94881 & -73.73262 & $\begin{array}{l}\text { real-time } \\
\text { RDG }\end{array}$ & storm tide & 10.16 & $\begin{array}{c}\text { 10/30/2012 } \\
\text { 02:00:00 }\end{array}$ \\
\hline $\begin{array}{l}\text { SSS-NY-KIN- } \\
\text { 001WL }\end{array}$ & New York & Kings & 40.58000 & -74.01161 & water level & storm tide & 13.32 & $\begin{array}{c}\text { 10/30/2012 } \\
00: 23: 30\end{array}$ \\
\hline $\begin{array}{l}\text { SSS-NY-KIN- } \\
\text { 003WL }\end{array}$ & New York & Kings & 40.67688 & -73.98984 & water level & storm tide & 11.08 & $\begin{array}{c}\text { 10/30/2012 } \\
01: 04: 30\end{array}$ \\
\hline $\begin{array}{l}\text { SSS-NY-NAS- } \\
001 \mathrm{WL}\end{array}$ & New York & Nassau & 40.87791 & -73.53057 & water level & storm tide & 10.12 & $\begin{array}{c}10 / 30 / 2012 \\
01: 49: 00\end{array}$ \\
\hline $\begin{array}{l}\text { SSS-NY-NAS- } \\
\text { 004WL }\end{array}$ & New York & Nassau & 40.58275 & -73.64068 & water level & storm tide & 17.48 & $\begin{array}{c}\text { 10/30/2012 } \\
\text { 00:00:00 }\end{array}$ \\
\hline $\begin{array}{l}\text { SSS-NY-NAS- } \\
004 \mathrm{WV}\end{array}$ & New York & Nassau & 40.58275 & -73.64068 & wave height & $\begin{array}{l}\text { wave } \\
\text { height }\end{array}$ & 16.54 & $\begin{array}{c}10 / 29 / 2012 \\
23: 51: 46\end{array}$ \\
\hline $\begin{array}{l}\text { SSS-NY-NAS- } \\
\text { 005WL }\end{array}$ & New York & Nassau & 40.65238 & -73.45850 & water level & storm tide & 7.98 & $\begin{array}{c}\text { 10/30/2012 } \\
01: 24: 00\end{array}$ \\
\hline $\begin{array}{l}\text { SSS-NY-NEW- } \\
\text { 001WL }\end{array}$ & New York & New York & 40.87757 & -73.92633 & water level & storm tide & 9.50 & $\begin{array}{c}10 / 30 / 2012 \\
02: 07: 30\end{array}$ \\
\hline $\begin{array}{l}\text { SSS-NY-QUE- } \\
001 \mathrm{WL}\end{array}$ & New York & Queens & 40.76229 & -73.85828 & water level & storm tide & 10.35 & $\begin{array}{c}10 / 30 / 2012 \\
02: 06: 30\end{array}$ \\
\hline $\begin{array}{l}\text { SSS-NY-QUE- } \\
\text { 002WL }\end{array}$ & New York & Queens & 40.64533 & -73.83638 & water level & storm tide & 11.16 & $\begin{array}{c}10 / 30 / 2012 \\
01: 23: 00\end{array}$ \\
\hline $\begin{array}{l}\text { SSS-NY-QUE- } \\
\text { 004WL }\end{array}$ & New York & Queens & 40.79651 & -73.82879 & water level & storm tide & 10.57 & $\begin{array}{c}\text { 10/30/2012 } \\
02: 06: 00\end{array}$ \\
\hline $\begin{array}{l}\text { SSS-NY-QUE- } \\
\text { 005WL }\end{array}$ & New York & Queens & 40.60615 & -73.82265 & water level & storm tide & 10.38 & $\begin{array}{c}10 / 30 / 2012 \\
01: 18: 30\end{array}$ \\
\hline $\begin{array}{l}\text { SSS-NY-RIC- } \\
\text { 001WL }\end{array}$ & New York & Richmond & 40.59388 & -74.05985 & water level & storm tide & 15.02 & $\begin{array}{c}10 / 30 / 2012 \\
00: 23: 30\end{array}$ \\
\hline $\begin{array}{l}\text { SSS-NY-RIC- } \\
001 \mathrm{WV}\end{array}$ & New York & Richmond & 40.59388 & -74.05985 & wave height & $\begin{array}{l}\text { wave } \\
\text { height }\end{array}$ & 15.06 & $\begin{array}{c}10 / 30 / 2012 \\
00: 59: 38\end{array}$ \\
\hline $\begin{array}{l}\text { SSS-NY-RIC- } \\
\text { 003WL }\end{array}$ & New York & Richmond & 40.50188 & -74.23034 & water level & storm tide & 16.00 & $\begin{array}{c}10 / 30 / 2012 \\
00: 38: 30\end{array}$ \\
\hline
\end{tabular}


Table 3. Hurricane Sandy peak storm-tide data for 165 U.S. Geological Survey temporarily deployed sites, by State.—Continued [NAVD 88, North American Vertical Datum of 1988; GMT, Greenwich Mean Time; RDG, rapid-deployment gage]

\begin{tabular}{|c|c|c|c|c|c|c|c|c|}
\hline $\begin{array}{c}\text { Site } \\
\text { identification }\end{array}$ & State & County & $\begin{array}{c}\text { Latitude } \\
\text { Decima }\end{array}$ & Longitude & Site type & $\begin{array}{c}\text { Type } \\
\text { of data } \\
\text { recorded }\end{array}$ & $\begin{array}{c}\text { Peak } \\
\text { storm-tide } \\
\text { elevation, } \\
\text { feet above } \\
\text { NAVD } 88\end{array}$ & $\begin{array}{c}\text { Peak } \\
\text { storm-tide } \\
\text { date and time } \\
\text { (GMT) }\end{array}$ \\
\hline $\begin{array}{l}\text { SSS-NY-RIC- } \\
004 \mathrm{WL}\end{array}$ & New York & Richmond & 40.54345 & -74.12768 & water level & storm tide & 13.22 & $\begin{array}{c}10 / 30 / 2012 \\
00: 52: 30\end{array}$ \\
\hline $\begin{array}{l}\text { SSS-NY-SUF- } \\
001 \mathrm{WL}\end{array}$ & New York & Suffolk & 41.01259 & -72.55828 & water level & storm tide & 7.86 & $\begin{array}{c}10 / 30 / 2012 \\
01: 47: 30\end{array}$ \\
\hline $\begin{array}{l}\text { SSS-NY-SUF- } \\
002 \mathrm{WL}\end{array}$ & New York & Suffolk & 40.96438 & -72.86320 & water level & storm tide & 8.48 & $\begin{array}{c}\text { 10/30/2012 } \\
01: 50: 00\end{array}$ \\
\hline $\begin{array}{l}\text { SSS-NY-SUF- } \\
\text { 003WL }\end{array}$ & New York & Suffolk & 40.94617 & -73.07227 & water level & storm tide & 8.81 & $\begin{array}{c}10 / 30 / 2012 \\
02: 02: 00\end{array}$ \\
\hline $\begin{array}{l}\text { SSS-NY-SUF- } \\
\text { 004WL }\end{array}$ & New York & Suffolk & 40.78712 & -72.75025 & water level & storm tide & 6.83 & $\begin{array}{c}10 / 30 / 2012 \\
01: 31: 30\end{array}$ \\
\hline $\begin{array}{l}\text { SSS-NY-SUF- } \\
\text { 005WL }\end{array}$ & New York & Suffolk & 40.91608 & -72.63774 & water level & storm tide & 7.86 & $\begin{array}{c}10 / 29 / 2012 \\
20: 33: 00\end{array}$ \\
\hline $\begin{array}{l}\text { SSS-NY-SUF- } \\
\text { 006WL }\end{array}$ & New York & Suffolk & 40.84887 & -72.50285 & water level & storm tide & 7.32 & $\begin{array}{c}10 / 30 / 2012 \\
01: 25: 30\end{array}$ \\
\hline $\begin{array}{l}\text { SSS-NY-SUF- } \\
\text { 008WL }\end{array}$ & New York & Suffolk & 40.89331 & -72.50300 & water level & storm tide & 6.53 & $\begin{array}{c}10 / 30 / 2012 \\
01: 59: 30\end{array}$ \\
\hline $\begin{array}{l}\text { SSS-NY-SUF- } \\
\text { 009WL }\end{array}$ & New York & Suffolk & 41.00197 & -72.29030 & water level & storm tide & 6.33 & $\begin{array}{c}10 / 30 / 2012 \\
01: 50: 30\end{array}$ \\
\hline $\begin{array}{l}\text { SSS-NY-SUF- } \\
\text { 011WL }\end{array}$ & New York & Suffolk & 40.90048 & -73.35304 & water level & storm tide & 9.48 & $\begin{array}{c}10 / 30 / 2012 \\
02: 18: 30\end{array}$ \\
\hline $\begin{array}{l}\text { SSS-NY-SUF- } \\
\text { 014WL }\end{array}$ & New York & Suffolk & 40.99070 & -72.47074 & water level & storm tide & 7.44 & $\begin{array}{c}\text { 10/30/2012 } \\
01: 13: 00\end{array}$ \\
\hline $\begin{array}{l}\text { SSS-NY-SUF- } \\
\text { 015WL }\end{array}$ & New York & Suffolk & 41.10104 & -72.36144 & water level & storm tide & 6.40 & $\begin{array}{c}10 / 30 / 2012 \\
01: 28: 00\end{array}$ \\
\hline $\begin{array}{l}\text { SSS-NY-SUF- } \\
\text { 017WL }\end{array}$ & New York & Suffolk & 40.64316 & -73.15750 & water level & storm tide & 13.41 & $\begin{array}{c}10 / 30 / 2012 \\
00: 12: 30\end{array}$ \\
\hline $\begin{array}{l}\text { SSS-NY-SUF- } \\
017 \mathrm{WV}\end{array}$ & New York & Suffolk & 40.64316 & -73.15750 & wave height & $\begin{array}{l}\text { wave } \\
\text { height }\end{array}$ & 15.93 & $\begin{array}{c}10 / 29 / 2012 \\
23: 16: 42\end{array}$ \\
\hline $\begin{array}{l}\text { SSS-NY-SUF- } \\
\text { 018WL }\end{array}$ & New York & Suffolk & 40.63473 & -73.20216 & water level & storm tide & 4.09 & $\begin{array}{c}\text { 10/30/2012 } \\
00: 26: 00\end{array}$ \\
\hline $\begin{array}{l}\text { SSS-NY-SUF- } \\
\text { 019WL }\end{array}$ & New York & Suffolk & 40.65932 & -73.26486 & water level & storm tide & 5.57 & $\begin{array}{c}10 / 30 / 2012 \\
01: 23: 30\end{array}$ \\
\hline $\begin{array}{l}\text { SSS-NY-SUF- } \\
\text { 021WL }\end{array}$ & New York & Suffolk & 40.74918 & -73.01338 & water level & storm tide & 6.73 & $\begin{array}{c}\text { 10/30/2012 } \\
03: 18: 00\end{array}$ \\
\hline $\begin{array}{l}\text { SSS-NY-SUF- } \\
022 \mathrm{WL}\end{array}$ & New York & Suffolk & 40.68523 & -73.27990 & water level & storm tide & 6.79 & $\begin{array}{c}10 / 30 / 2012 \\
01: 36: 30\end{array}$ \\
\hline $\begin{array}{l}\text { SSS-NY-SUF- } \\
\text { 023WL }\end{array}$ & New York & Suffolk & 40.94428 & -72.18910 & water level & none & none & lost \\
\hline $\begin{array}{l}\text { SSS-NY-SUF- } \\
023 \mathrm{WV}\end{array}$ & New York & Suffolk & 40.94428 & -72.18910 & wave height & $\begin{array}{l}\text { wave } \\
\text { height }\end{array}$ & 13.39 & $\begin{array}{c}10 / 29 / 2012 \\
23: 19: 44\end{array}$ \\
\hline $\begin{array}{l}\text { SSS-NY-SUF- } \\
\text { 024WL }\end{array}$ & New York & Suffolk & 41.07319 & -71.93438 & water level & storm tide & 6.08 & $\begin{array}{c}10 / 29 / 2012 \\
23: 13: 00\end{array}$ \\
\hline $\begin{array}{l}\text { SSS-NY-SUF- } \\
\text { 026WL }\end{array}$ & New York & Suffolk & 40.74687 & -72.85550 & water level & storm tide & 5.68 & $\begin{array}{c}10 / 30 / 2012 \\
01: 20: 30\end{array}$ \\
\hline
\end{tabular}


Table 3. Hurricane Sandy peak storm-tide data for 165 U.S. Geological Survey temporarily deployed sites, by State.-Continued [NAVD 88, North American Vertical Datum of 1988; GMT, Greenwich Mean Time; RDG, rapid-deployment gage]

\begin{tabular}{|c|c|c|c|c|c|c|c|c|}
\hline $\begin{array}{c}\text { Site } \\
\text { identification }\end{array}$ & State & County & $\begin{array}{l}\text { Latitude } \\
\text { Decima }\end{array}$ & Longitude & Site type & $\begin{array}{c}\text { Type } \\
\text { of data } \\
\text { recorded }\end{array}$ & $\begin{array}{c}\text { Peak } \\
\text { storm-tide } \\
\text { elevation, } \\
\text { feet above } \\
\text { NAVD } 88\end{array}$ & $\begin{array}{c}\text { Peak } \\
\text { storm-tide } \\
\text { date and time } \\
\text { (GMT) }\end{array}$ \\
\hline $\begin{array}{l}\text { SSS-NY-SUF- } \\
\text { 027WL }\end{array}$ & New York & Suffolk & 40.74760 & -73.15039 & water level & storm tide & 6.06 & $\begin{array}{c}10 / 30 / 2012 \\
03: 57: 30\end{array}$ \\
\hline $\begin{array}{l}\text { SSS-NY-WES- } \\
\text { 003WL }\end{array}$ & New York & Westchester & 40.89039 & -73.78172 & water level & storm tide & 10.44 & $\begin{array}{c}\text { 10/30/2012 } \\
01: 59: 30\end{array}$ \\
\hline $\begin{array}{l}\text { SSS-PA-DEL- } \\
\text { 003WL }\end{array}$ & Pennsylvania & Delaware & 39.83280 & -75.37609 & water level & storm tide & 7.01 & $\begin{array}{c}\text { 10/30/2012 } \\
07: 02: 00\end{array}$ \\
\hline $\begin{array}{l}\text { SSS-PA-DEL- } \\
005 \mathrm{WL}\end{array}$ & Pennsylvania & Delaware & 39.85918 & -75.30225 & water level & storm tide & 7.11 & $\begin{array}{c}\text { 10/30/2012 } \\
07: 29: 00\end{array}$ \\
\hline $\begin{array}{c}\text { SSS-PA-PHI- } \\
\text { 013WL }\end{array}$ & Pennsylvania & Philadelphia & 40.03425 & -75.00025 & water level & none & none & $\begin{array}{l}\text { no surge } \\
\text { recorded }\end{array}$ \\
\hline $\begin{array}{l}\text { SSS-PA-PHI- } \\
\text { 014WL }\end{array}$ & Pennsylvania & Philadelphia & 39.93389 & -75.20825 & water level & storm tide & 7.44 & $\begin{array}{c}10 / 30 / 2012 \\
07: 29: 30\end{array}$ \\
\hline $\begin{array}{l}\text { SSS-PA-PHI- } \\
\text { 016WL }\end{array}$ & Pennsylvania & Philadelphia & 39.88758 & -75.16422 & water level & storm tide & 7.52 & $\begin{array}{c}10 / 30 / 2012 \\
07: 52: 30\end{array}$ \\
\hline 412656071265946 & Rhode Island & Washington & 41.44911 & -71.44985 & $\begin{array}{l}\text { real-time } \\
\text { RDG }\end{array}$ & storm tide & 6.02 & $\begin{array}{c}\text { 10/30/2012 } \\
00: 15: 00\end{array}$ \\
\hline $\begin{array}{l}\text { SSS-RI-BRI- } \\
\text { 013WL }\end{array}$ & Rhode Island & Bristol & 41.72614 & -71.28590 & water level & storm tide & 6.27 & $\begin{array}{c}10 / 29 / 2012 \\
23: 12: 00\end{array}$ \\
\hline $\begin{array}{l}\text { SSS-RI-WAS- } \\
\text { 001WL }\end{array}$ & Rhode Island & Washington & 41.31029 & -71.85914 & water level & storm tide & 5.86 & $\begin{array}{c}10 / 29 / 2012 \\
23: 52: 30\end{array}$ \\
\hline $\begin{array}{l}\text { SSS-RI-WAS- } \\
\text { 003WL }\end{array}$ & Rhode Island & Washington & 41.36451 & -71.60520 & water level & storm tide & 8.43 & $\begin{array}{c}\text { 10/30/2012 } \\
00: 27: 00\end{array}$ \\
\hline $\begin{array}{l}\text { SSS-RI-WAS- } \\
\text { 005WL }\end{array}$ & Rhode Island & Washington & 41.33482 & -71.76663 & water level & storm tide & 6.39 & $\begin{array}{c}10 / 30 / 2012 \\
00: 31: 30\end{array}$ \\
\hline $\begin{array}{l}\text { SSS-RI-WAS- } \\
\text { 007WL }\end{array}$ & Rhode Island & Washington & 41.38102 & -71.64473 & water level & storm tide & 3.97 & $\begin{array}{c}10 / 30 / 2012 \\
02: 01: 00\end{array}$ \\
\hline $\begin{array}{l}\text { SSS-RI-WAS- } \\
\text { 008WL }\end{array}$ & Rhode Island & Washington & 41.37726 & -71.51472 & water level & storm tide & 6.59 & $\begin{array}{c}10 / 29 / 2012 \\
23: 59: 30\end{array}$ \\
\hline $\begin{array}{l}\text { SSS-RI-WAS- } \\
\text { 012WL }\end{array}$ & Rhode Island & Washington & 41.52809 & -71.41637 & water level & storm tide & 6.35 & $\begin{array}{c}10 / 29 / 2012 \\
22: 23: 00\end{array}$ \\
\hline $\begin{array}{l}\text { SSS-VA-ACC- } \\
001 \mathrm{WL}\end{array}$ & Virginia & Accomack & 37.90318 & -75.40668 & water level & storm tide & 5.00 & $\begin{array}{c}10 / 29 / 2012 \\
12: 51: 00\end{array}$ \\
\hline $\begin{array}{l}\text { SSS-VA-ACC- } \\
\text { 002WL }\end{array}$ & Virginia & Accomack & 37.73291 & -75.58983 & water level & storm tide & 5.98 & $\begin{array}{c}10 / 29 / 2012 \\
11: 41: 00\end{array}$ \\
\hline
\end{tabular}


Table 3. Hurricane Sandy peak storm-tide data for 165 U.S. Geological Survey temporarily deployed sites, by State.-Continued [NAVD 88, North American Vertical Datum of 1988; GMT, Greenwich Mean Time; RDG, rapid-deployment gage]

\begin{tabular}{|c|c|c|c|c|c|c|c|c|}
\hline $\begin{array}{c}\text { Site } \\
\text { identification }\end{array}$ & State & County & \multicolumn{2}{|c|}{ Decimal degrees } & Site type & $\begin{array}{c}\text { Type } \\
\text { of data } \\
\text { recorded }\end{array}$ & $\begin{array}{c}\text { Peak } \\
\text { storm-tide } \\
\text { elevation, } \\
\text { feet above } \\
\text { NAVD } 88\end{array}$ & $\begin{array}{c}\text { Peak } \\
\text { storm-tide } \\
\text { date and time } \\
\text { (GMT) }\end{array}$ \\
\hline $\begin{array}{l}\text { SSS-VA-ACC- } \\
003 \mathrm{WL}\end{array}$ & Virginia & Accomack & 37.72195 & -75.78580 & water level & storm tide & 5.38 & $\begin{array}{c}10 / 29 / 2012 \\
16: 48: 00\end{array}$ \\
\hline $\begin{array}{l}\text { SSS-VA-MAT- } \\
\text { 001WL }\end{array}$ & Virginia & Mathews & 37.49278 & -76.31000 & water level & storm tide & 3.54 & $\begin{array}{c}10 / 29 / 2012 \\
14: 16: 00\end{array}$ \\
\hline $\begin{array}{l}\text { SSS-VA-NOR- } \\
\text { 001WL }\end{array}$ & Virginia & Northampton & 37.44528 & -75.84186 & water level & storm tide & 5.46 & $\begin{array}{c}10 / 29 / 2012 \\
13: 05: 00\end{array}$ \\
\hline $\begin{array}{l}\text { SSS-VA-NOR- } \\
\text { 003WL }\end{array}$ & Virginia & Northampton & 37.26492 & -76.01628 & water level & storm tide & 5.02 & $\begin{array}{c}10 / 29 / 2012 \\
13: 36: 00\end{array}$ \\
\hline $\begin{array}{l}\text { SSS-VA-VAB- } \\
\text { 001WL }\end{array}$ & Virginia & $\begin{array}{r}\text { Virginia } \\
\text { Beach }\end{array}$ & 36.90683 & -76.08825 & water level & storm tide & 5.49 & $\begin{array}{c}10 / 29 / 2012 \\
12: 56: 00\end{array}$ \\
\hline $\begin{array}{l}\text { SSS-VA-YOR- } \\
003 \mathrm{WL}\end{array}$ & Virginia & Poquoson & 37.11061 & -76.31944 & water level & storm tide & 5.08 & $\begin{array}{c}10 / 29 / 2012 \\
12: 48: 00\end{array}$ \\
\hline
\end{tabular}


Table 4. Hurricane Sandy peak storm-tide data recorded at U.S. Geological Survey permanent monitoring sites, by State.

[NAVD 88, North American Vertical Datum of 1988; NGVD 29, National Geodetic Vertical Datum of 1929; GMT, Greenwich Mean Time]

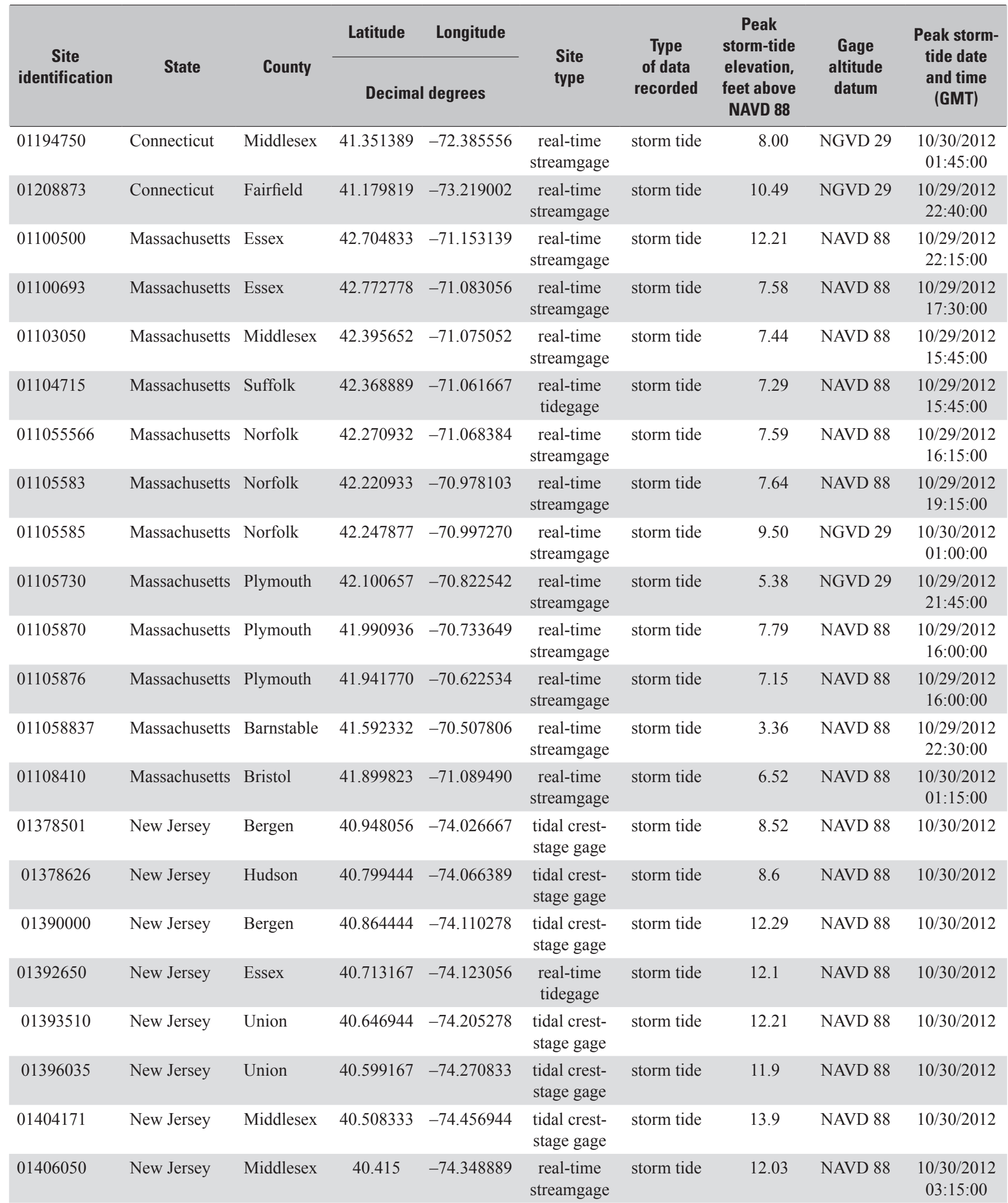


Table 4. Hurricane Sandy peak storm-tide data recorded at U.S. Geological Survey permanent monitoring sites, by State.-Continued [NAVD 88, North American Vertical Datum of 1988; NGVD 29, National Geodetic Vertical Datum of 1929; GMT, Greenwich Mean Time]

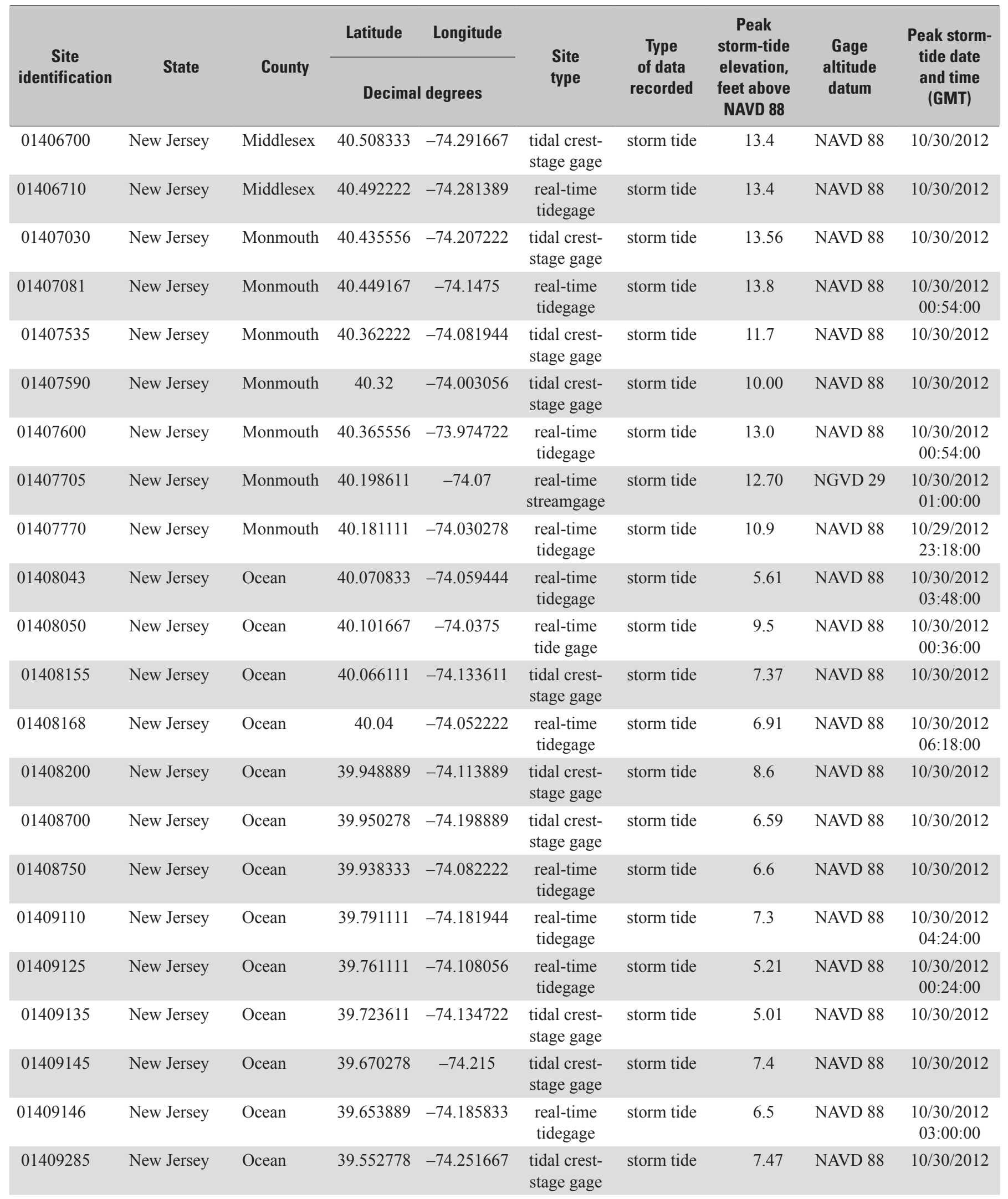


Table 4. Hurricane Sandy peak storm-tide data recorded at U.S. Geological Survey permanent monitoring sites, by State.—Continued [NAVD 88, North American Vertical Datum of 1988; NGVD 29, National Geodetic Vertical Datum of 1929; GMT, Greenwich Mean Time]

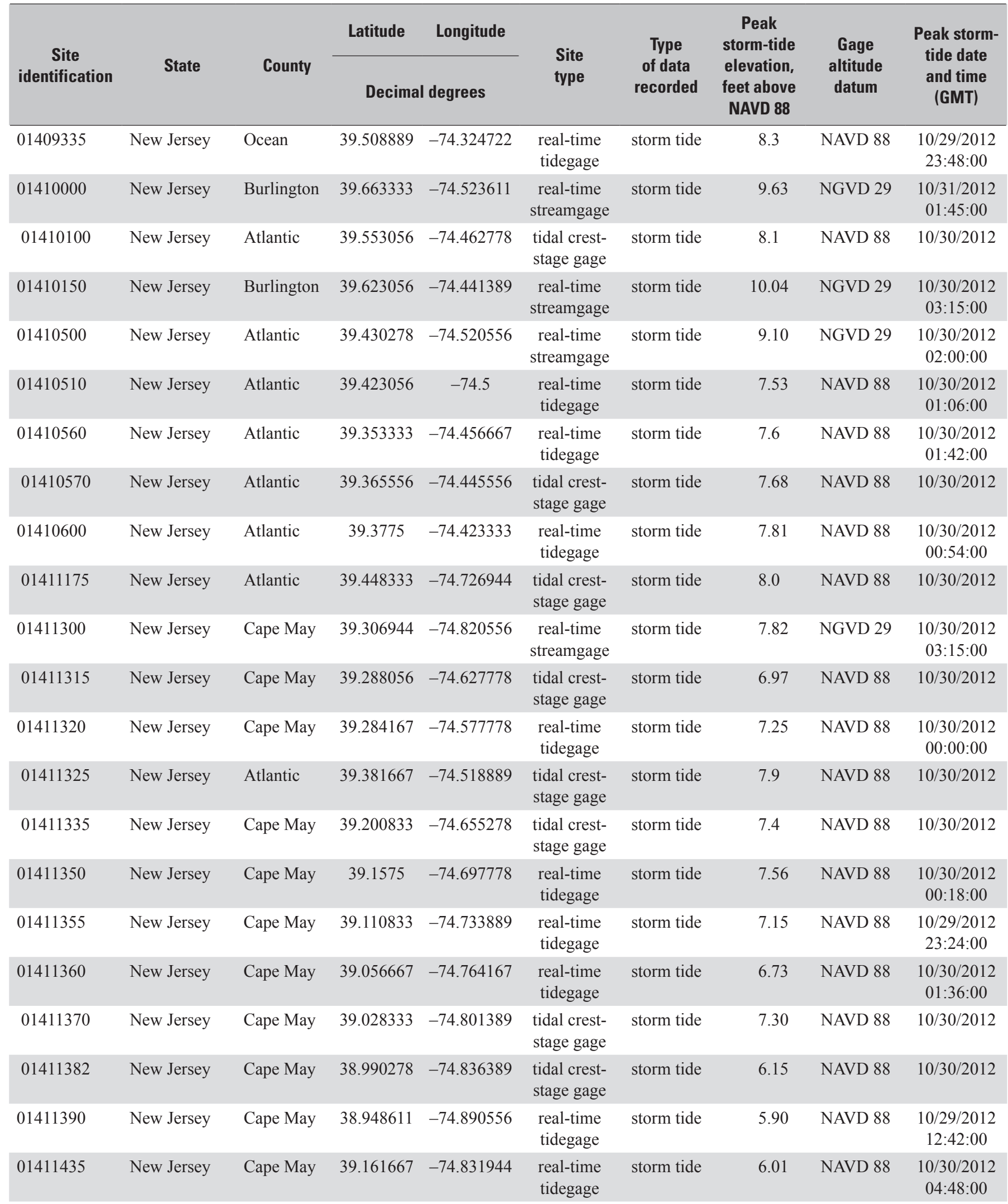


Table 4. Hurricane Sandy peak storm-tide data recorded at U.S. Geological Survey permanent monitoring sites, by State.-Continued [NAVD 88, North American Vertical Datum of 1988; NGVD 29, National Geodetic Vertical Datum of 1929; GMT, Greenwich Mean Time]

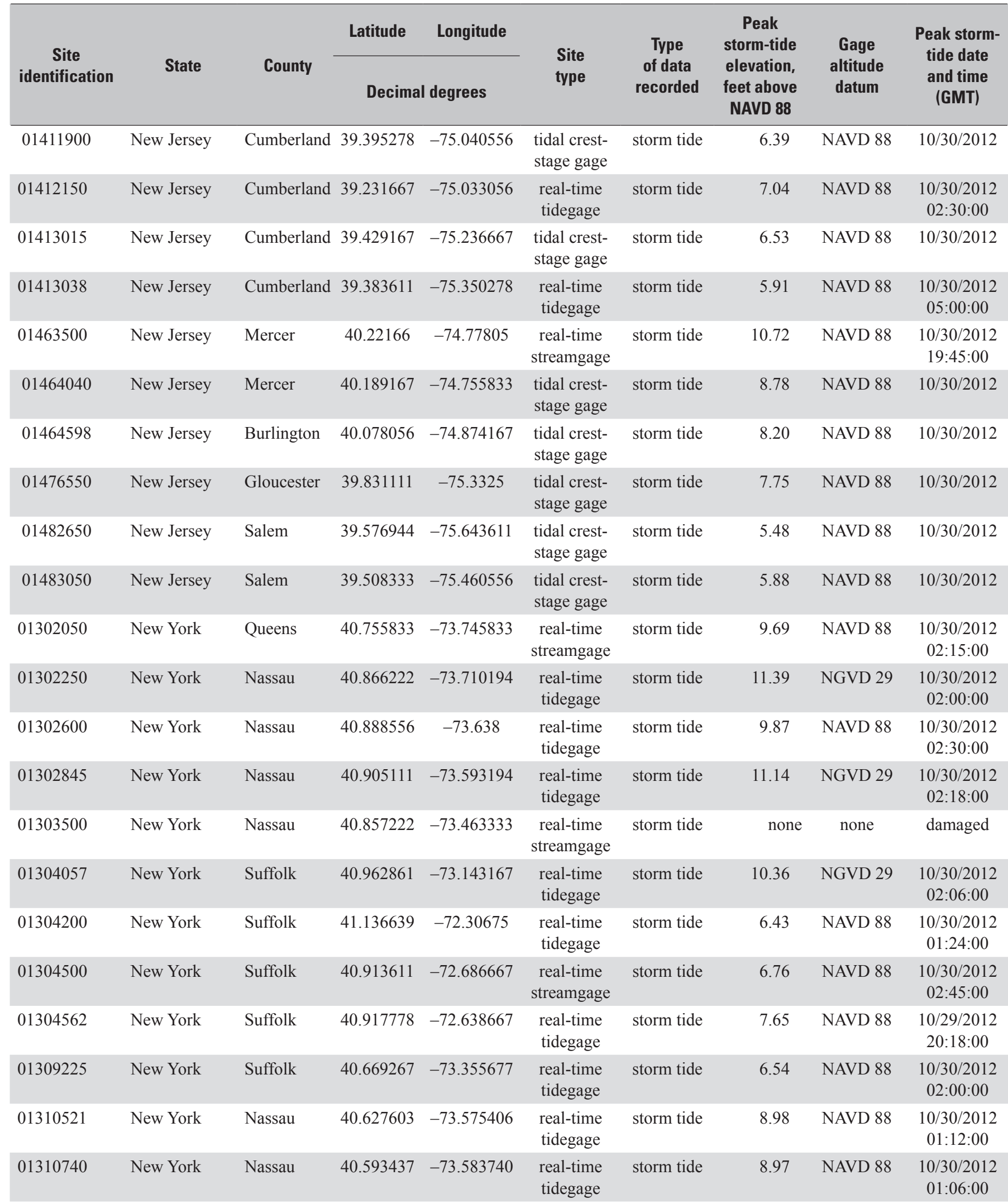


Table 4. Hurricane Sandy peak storm-tide data recorded at U.S. Geological Survey permanent monitoring sites, by State.-Continued [NAVD 88, North American Vertical Datum of 1988; NGVD 29, National Geodetic Vertical Datum of 1929; GMT, Greenwich Mean Time]

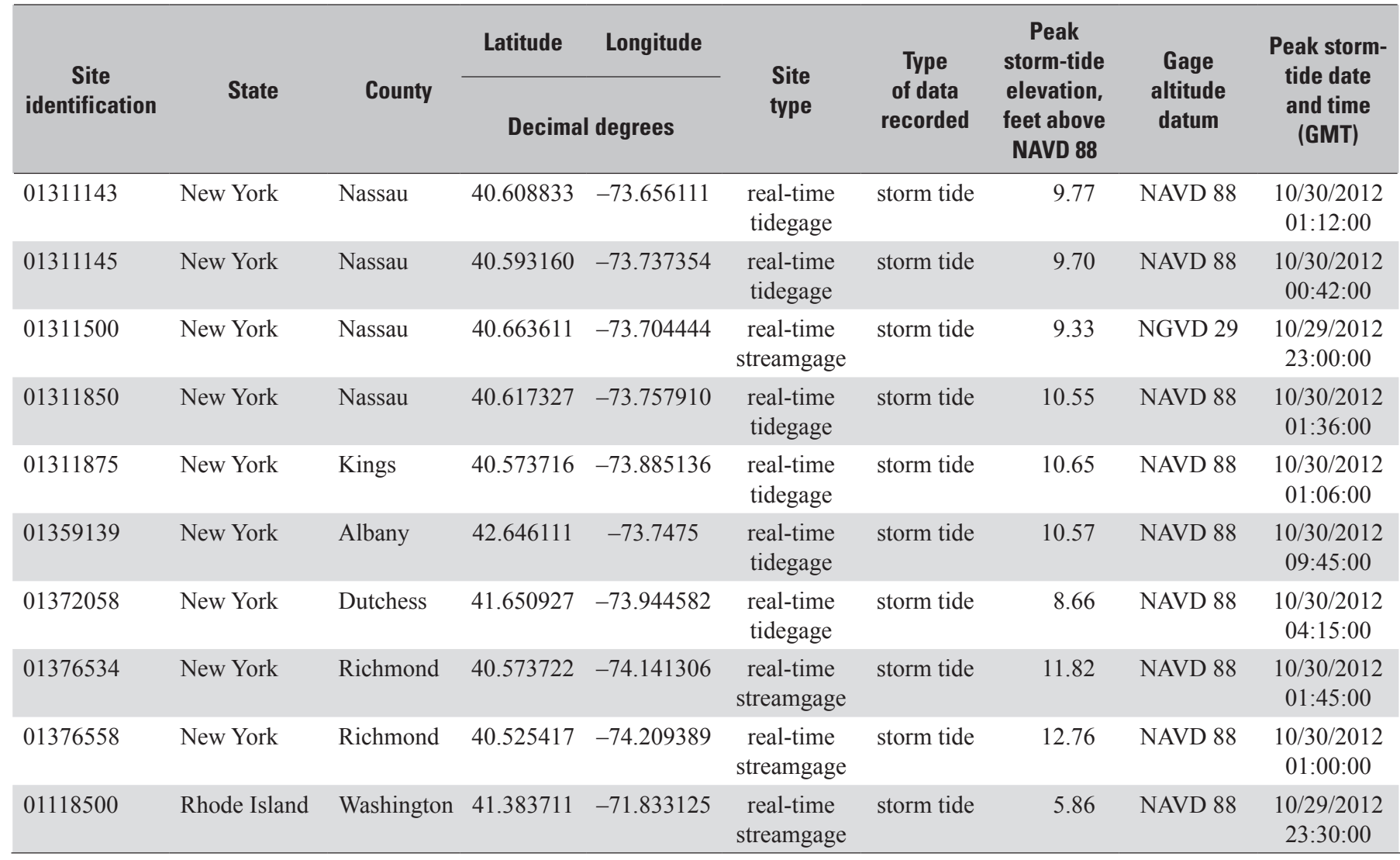


Table 5. Hurricane Sandy peak storm-tide data recorded at National Oceanic and Atmospheric Administration permanent monitoring sites, by State (NOAA, 2012).

[NAVD 88, North American Vertical Datum of 1988; GMT, Greenwich Mean Time]

\begin{tabular}{|c|c|c|c|c|c|c|c|c|}
\hline $\begin{array}{c}\text { Site } \\
\text { identification }\end{array}$ & State & County & $\begin{array}{c}\text { Latitude } \\
\text { Decim }\end{array}$ & Longitude & $\begin{array}{l}\text { Site } \\
\text { type }\end{array}$ & $\begin{array}{c}\text { Type } \\
\text { of data } \\
\text { recorded }\end{array}$ & $\begin{array}{c}\text { Peak } \\
\text { storm-tide } \\
\text { elevation, } \\
\text { feet above } \\
\text { NAVD } 88\end{array}$ & $\begin{array}{c}\text { Peak } \\
\text { storm-tide } \\
\text { date } \\
\text { and time } \\
\text { (GMT) }\end{array}$ \\
\hline 8461490 & Connecticut & New London & 41.36000 & -72.09000 & $\begin{array}{l}\text { real-time } \\
\text { tide gage }\end{array}$ & storm tide & 6.16 & $\begin{array}{c}10 / 30 / 2012 \\
0: 12\end{array}$ \\
\hline 8467150 & Connecticut & Fairfield & 41.17333 & -73.18167 & $\begin{array}{l}\text { real-time } \\
\text { tide gage }\end{array}$ & storm tide & 9.30 & $\begin{array}{c}10 / 30 / 2012 \\
2: 06\end{array}$ \\
\hline 8551910 & Delaware & New Castle & 39.55833 & -75.57333 & $\begin{array}{l}\text { real-time } \\
\text { tide gage }\end{array}$ & storm tide & 6.13 & $\begin{array}{c}10 / 30 / 2012 \\
5: 42\end{array}$ \\
\hline 8557380 & Delaware & Sussex & 38.78167 & -75.12000 & $\begin{array}{l}\text { real-time } \\
\text { tide gage }\end{array}$ & storm tide & 6.08 & $\begin{array}{c}10 / 29 / 2012 \\
13: 00\end{array}$ \\
\hline 8570283 & Maryland & Worcester & 38.32833 & -75.09167 & $\begin{array}{l}\text { real-time } \\
\text { tide gage }\end{array}$ & storm tide & 4.42 & $\begin{array}{c}10 / 29 / 2012 \\
13: 42\end{array}$ \\
\hline 8571421 & Maryland & Dorchester & 38.22000 & -76.03833 & $\begin{array}{l}\text { real-time } \\
\text { tide gage }\end{array}$ & storm tide & 3.05 & $\begin{array}{c}10 / 30 / 2012 \\
18: 36\end{array}$ \\
\hline 8571892 & Maryland & Dorchester & 38.57333 & -76.06833 & $\begin{array}{l}\text { real-time } \\
\text { tide gage }\end{array}$ & storm tide & 3.44 & $\begin{array}{c}10 / 29 / 2012 \\
21: 30\end{array}$ \\
\hline 8574680 & Maryland & Baltimore & 39.26667 & -76.57833 & $\begin{array}{l}\text { real-time } \\
\text { tide gage }\end{array}$ & storm tide & 3.83 & $\begin{array}{c}10 / 30 / 2012 \\
10: 36\end{array}$ \\
\hline 8575512 & Maryland & Anne Arundel & 38.98333 & -76.48000 & $\begin{array}{l}\text { real-time } \\
\text { tide gage }\end{array}$ & storm tide & 3.11 & $\begin{array}{c}10 / 30 / 2012 \\
12: 54\end{array}$ \\
\hline 8577330 & Maryland & Calvert & 38.31667 & -76.45167 & $\begin{array}{l}\text { real-time } \\
\text { tide gage }\end{array}$ & storm tide & none & damaged \\
\hline 8443970 & Massachusetts & Suffolk & 42.35333 & -71.05333 & $\begin{array}{l}\text { real-time } \\
\text { tide gage }\end{array}$ & storm tide & 7.42 & $\begin{array}{c}10 / 29 / 2012 \\
15: 48\end{array}$ \\
\hline 8447435 & Massachusetts & Barnstable & 41.68833 & -69.95000 & $\begin{array}{l}\text { real-time } \\
\text { tide gage }\end{array}$ & storm tide & 5.87 & $\begin{array}{c}10 / 29 / 2012 \\
16: 00\end{array}$ \\
\hline 8447930 & Massachusetts & Barnstable & 41.52333 & -70.67167 & $\begin{array}{l}\text { real-time } \\
\text { tide gage }\end{array}$ & storm tide & 4.44 & $\begin{array}{c}10 / 29 / 2012 \\
22: 18\end{array}$ \\
\hline 8423898 & New Hampshire & Rockingham & 43.07167 & -70.71167 & $\begin{array}{l}\text { real-time } \\
\text { tide gage }\end{array}$ & storm tide & 6.41 & $\begin{array}{c}10 / 29 / 2012 \\
15: 36\end{array}$ \\
\hline 8531680 & New Jersey & Monmouth & 40.46667 & -74.00833 & $\begin{array}{l}\text { real-time } \\
\text { tide gage }\end{array}$ & storm tide & none & damaged \\
\hline 8534720 & New Jersey & Atlantic & 39.35500 & -74.41833 & $\begin{array}{l}\text { real-time } \\
\text { tide gage }\end{array}$ & storm tide & 6.28 & $\begin{array}{c}10 / 30 / 2012 \\
0: 24\end{array}$ \\
\hline 8536110 & New Jersey & Cape May & 38.96833 & -74.96000 & $\begin{array}{l}\text { real-time } \\
\text { tide gage }\end{array}$ & storm tide & 5.90 & $\begin{array}{c}10 / 29 / 2012 \\
13: 42\end{array}$ \\
\hline 8510560 & New York & Suffolk & 41.04833 & -71.96000 & $\begin{array}{l}\text { real-time } \\
\text { tide gage }\end{array}$ & storm tide & 5.55 & $\begin{array}{c}10 / 30 / 2012 \\
0: 12\end{array}$ \\
\hline 8518750 & New York & New York & 40.70000 & -74.01333 & $\begin{array}{l}\text { real-time } \\
\text { tide gage }\end{array}$ & storm tide & 11.28 & $\begin{array}{c}10 / 30 / 2012 \\
1: 24\end{array}$ \\
\hline 8545240 & Pennsylvania & Philadelphia & 39.93333 & -75.14167 & $\begin{array}{l}\text { real-time } \\
\text { tide gage }\end{array}$ & storm tide & 7.52 & $\begin{array}{c}10 / 30 / 2012 \\
8: 00\end{array}$ \\
\hline 8452660 & Rhode Island & Newport & 41.50500 & -71.32667 & $\begin{array}{l}\text { real-time } \\
\text { tide gage }\end{array}$ & storm tide & 6.13 & $\begin{array}{c}10 / 29 / 2012 \\
23: 00\end{array}$ \\
\hline 8454000 & Rhode Island & Providence & 41.80667 & -71.40000 & $\begin{array}{l}\text { real-time } \\
\text { tide gage }\end{array}$ & storm tide & 6.89 & $\begin{array}{c}10 / 29 / 2012 \\
23: 30\end{array}$ \\
\hline 8632200 & Virginia & Northampton & 37.16500 & -75.98833 & $\begin{array}{l}\text { real-time } \\
\text { tide gage }\end{array}$ & storm tide & 4.92 & $\begin{array}{c}10 / 29 / 2012 \\
12: 48\end{array}$ \\
\hline 8635750 & Virginia & $\begin{array}{l}\text { Northumber- } \\
\text { land }\end{array}$ & 37.99500 & -76.46333 & $\begin{array}{l}\text { real-time } \\
\text { tide gage }\end{array}$ & storm tide & 2.92 & $\begin{array}{c}10 / 28 / 2012 \\
18: 06\end{array}$ \\
\hline 8638610 & Virginia & Norfolk & 36.94667 & -76.33000 & $\begin{array}{l}\text { real-time } \\
\text { tide gage }\end{array}$ & storm tide & 5.17 & $\begin{array}{c}10 / 29 / 2012 \\
13: 18\end{array}$ \\
\hline
\end{tabular}


Table 6. Hurricane Sandy storm-tide high-water-mark data collected and surveyed by U.S. Geological Survey, by State. [NAVD 88, North American Vertical Datum of 1988; GMT, Greenwich Mean Time]

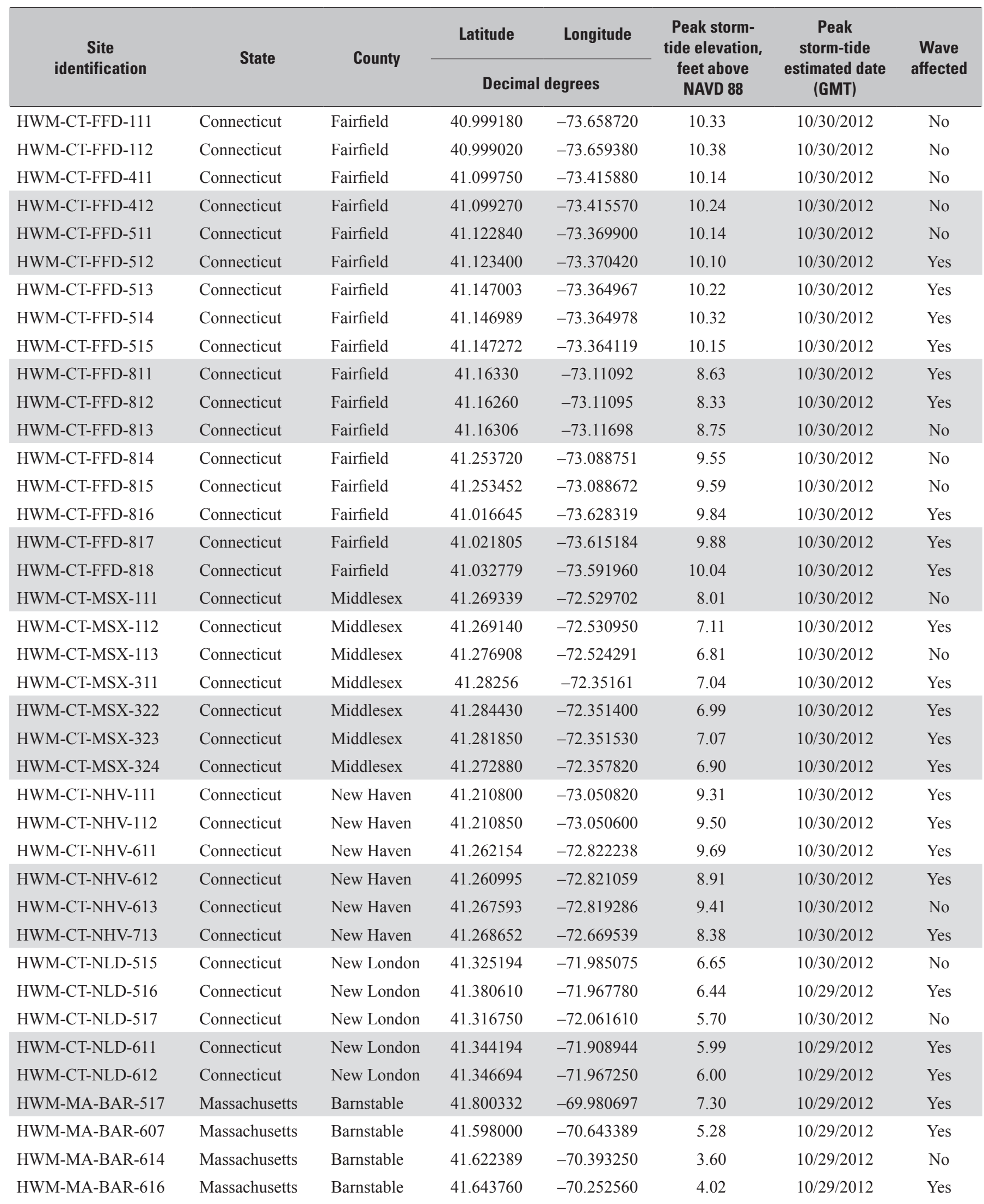


Table 6. Hurricane Sandy storm-tide high-water-mark data collected and surveyed by U.S. Geological Survey, by State.-Continued [NAVD 88, North American Vertical Datum of 1988; GMT, Greenwich Mean Time]

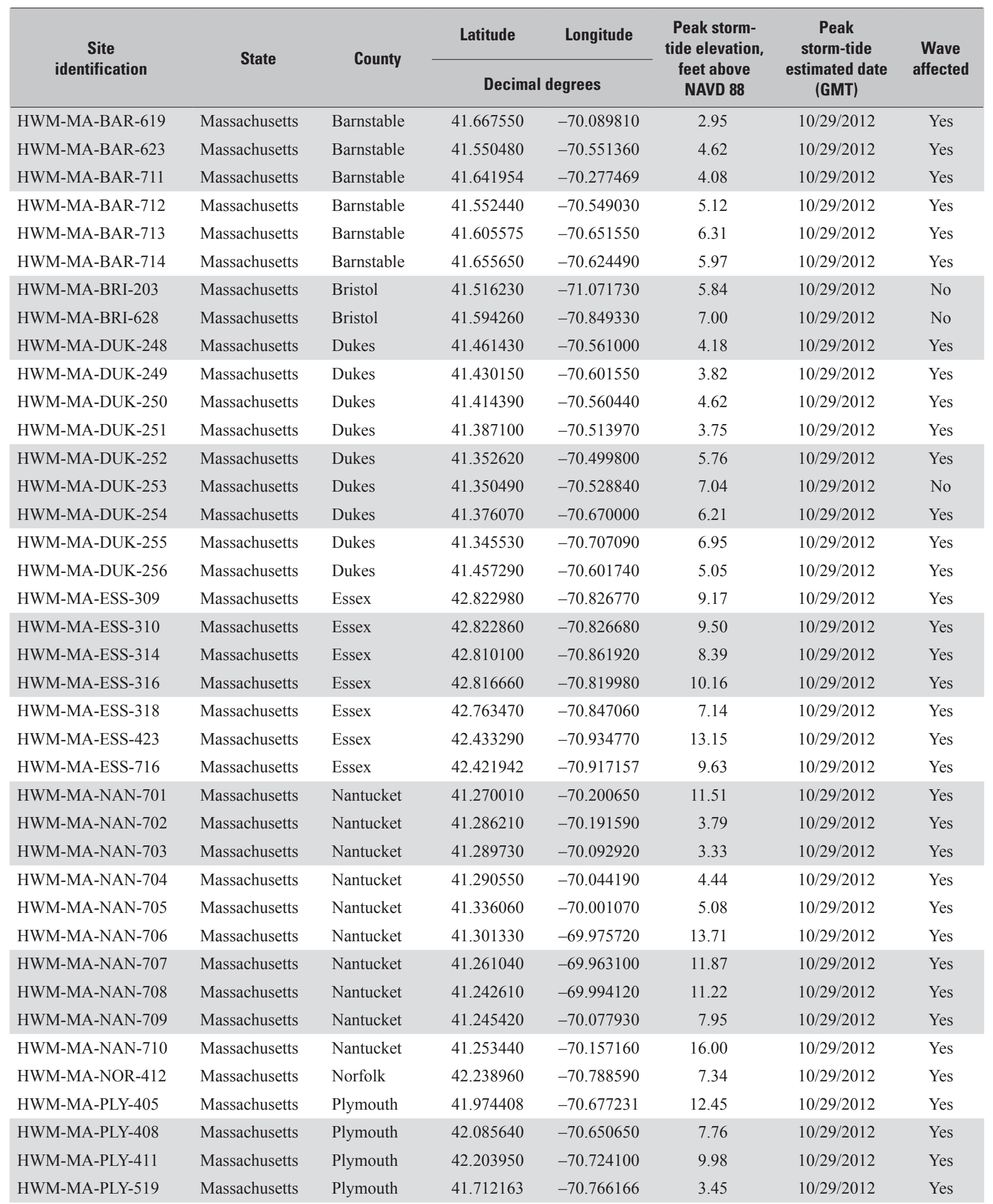


Table 6. Hurricane Sandy storm-tide high-water-mark data collected and surveyed by U.S. Geological Survey, by State.-Continued [NAVD 88, North American Vertical Datum of 1988; GMT, Greenwich Mean Time]

\begin{tabular}{|c|c|c|c|c|c|c|c|}
\hline \multirow{2}{*}{$\begin{array}{c}\text { Site } \\
\text { identification }\end{array}$} & \multirow{2}{*}{ State } & \multirow{2}{*}{ County } & Latitude & Longitude & \multirow{2}{*}{$\begin{array}{l}\text { Peak storm- } \\
\text { tide elevation, } \\
\text { feet above } \\
\text { NAVD } 88\end{array}$} & \multirow{2}{*}{$\begin{array}{c}\text { Peak } \\
\text { storm-tide } \\
\text { estimated date } \\
\text { (GMT) }\end{array}$} & \multirow{2}{*}{$\begin{array}{c}\text { Wave } \\
\text { affected }\end{array}$} \\
\hline & & & \multicolumn{2}{|c|}{ Decimal degrees } & & & \\
\hline HWM-MA-PLY-624 & Massachusetts & Plymouth & 41.747560 & -70.632260 & 5.02 & $10 / 29 / 2012$ & Yes \\
\hline HWM-MA-PLY-625 & Massachusetts & Plymouth & 41.657650 & -70.813800 & 5.94 & $10 / 29 / 2012$ & Yes \\
\hline HWM-NJ-ATL-006 & New Jersey & Atlantic & 39.553333 & -74.462778 & 8.18 & $10 / 30 / 2012$ & No \\
\hline HWM-NJ-ATL-007 & New Jersey & Atlantic & 39.553333 & -74.462778 & 8.11 & $10 / 30 / 2012$ & No \\
\hline HWM-NJ-ATL-100 & New Jersey & Atlantic & 39.430574 & -74.520151 & 7.62 & $10 / 29 / 2012$ & Yes \\
\hline HWM-NJ-ATL-101 & New Jersey & Atlantic & 39.422667 & -74.499918 & 7.39 & $10 / 29 / 2012$ & Yes \\
\hline HWM-NJ-ATL-105 & New Jersey & Atlantic & 39.390747 & -74.397936 & 7.64 & $10 / 29 / 2012$ & No \\
\hline HWM-NJ-ATL-106 & New Jersey & Atlantic & 39.377728 & -74.406839 & 7.79 & $10 / 29 / 2012$ & No \\
\hline HWM-NJ-ATL-107 & New Jersey & Atlantic & 39.415066 & -74.355190 & 8.00 & $10 / 29 / 2012$ & No \\
\hline HWM-NJ-ATL-108 & New Jersey & Atlantic & 39.405355 & -74.372788 & 7.75 & $10 / 29 / 2012$ & No \\
\hline HWM-NJ-ATL-232 & New Jersey & Atlantic & 39.623130 & -74.624340 & 7.38 & $10 / 29 / 2012$ & No \\
\hline HWM-NJ-ATL-300 & New Jersey & Atlantic & 39.357160 & -74.445450 & 7.31 & $10 / 29 / 2012$ & No \\
\hline HWM-NJ-ATL-301 & New Jersey & Atlantic & 39.361947 & -74.444327 & 7.22 & $10 / 30 / 2012$ & Yes \\
\hline HWM-NJ-ATL-302 & New Jersey & Atlantic & 39.348720 & -74.464810 & 7.71 & $10 / 29 / 2012$ & No \\
\hline HWM-NJ-BER-415 & New Jersey & Bergen & 40.842819 & -73.966186 & 9.44 & $10 / 30 / 2012$ & No \\
\hline HWM-NJ-BER-416 & New Jersey & Bergen & 40.842344 & -74.042028 & 7.43 & $10 / 30 / 2012$ & No \\
\hline HWM-NJ-BER-417 & New Jersey & Bergen & 40.785528 & -74.147158 & 11.82 & $10 / 30 / 2012$ & No \\
\hline HWM-NJ-BER-423 & New Jersey & Bergen & 40.816142 & -73.978506 & 9.46 & $10 / 30 / 2012$ & No \\
\hline HWM-NJ-BUR-233 & New Jersey & Burlington & 39.641960 & -74.649920 & 7.48 & $10 / 29 / 2012$ & No \\
\hline HWM-NJ-CPM-001 & New Jersey & Cape May & 38.957200 & -74.883020 & 5.72 & $10 / 29 / 2012$ & Yes \\
\hline HWM-NJ-CPM-002 & New Jersey & Cape May & 38.958800 & -74.854950 & 5.32 & $10 / 29 / 2012$ & Yes \\
\hline HWM-NJ-CPM-003 & New Jersey & Cape May & 38.964130 & -74.854380 & 5.88 & $10 / 29 / 2012$ & Yes \\
\hline HWM-NJ-CPM-004 & New Jersey & Cape May & 39.077440 & -74.802660 & 6.89 & $10 / 29 / 2012$ & No \\
\hline HWM-NJ-CPM-005 & New Jersey & Cape May & 39.060050 & -74.769220 & 6.73 & $10 / 29 / 2012$ & No \\
\hline HWM-NJ-CPM-006 & New Jersey & Cape May & 39.058580 & -74.756350 & 6.82 & $10 / 29 / 2012$ & No \\
\hline HWM-NJ-CPM-007 & New Jersey & Cape May & 39.082390 & -74.737680 & 7.77 & $10 / 29 / 2012$ & No \\
\hline HWM-NJ-CPM-008 & New Jersey & Cape May & 39.096630 & -74.724910 & 6.94 & $10 / 29 / 2012$ & No \\
\hline HWM-NJ-CPM-234 & New Jersey & Cape May & 39.116780 & -74.890870 & 6.72 & $10 / 29 / 2012$ & No \\
\hline
\end{tabular}


Table 6. Hurricane Sandy storm-tide high-water-mark data collected and surveyed by U.S. Geological Survey, by State.-Continued [NAVD 88, North American Vertical Datum of 1988; GMT, Greenwich Mean Time]

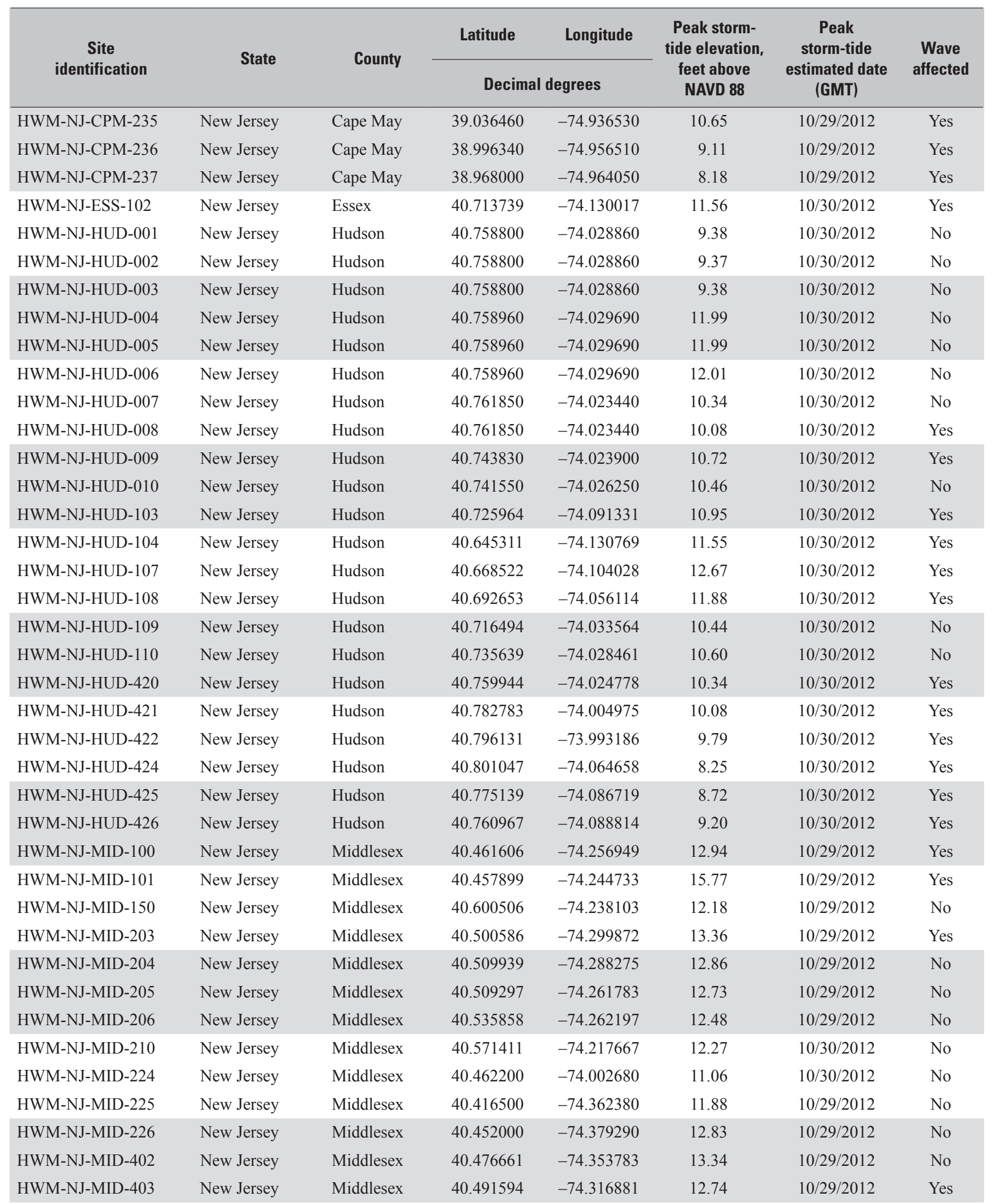


Table 6. Hurricane Sandy storm-tide high-water-mark data collected and surveyed by U.S. Geological Survey, by State.-Continued [NAVD 88, North American Vertical Datum of 1988; GMT, Greenwich Mean Time]

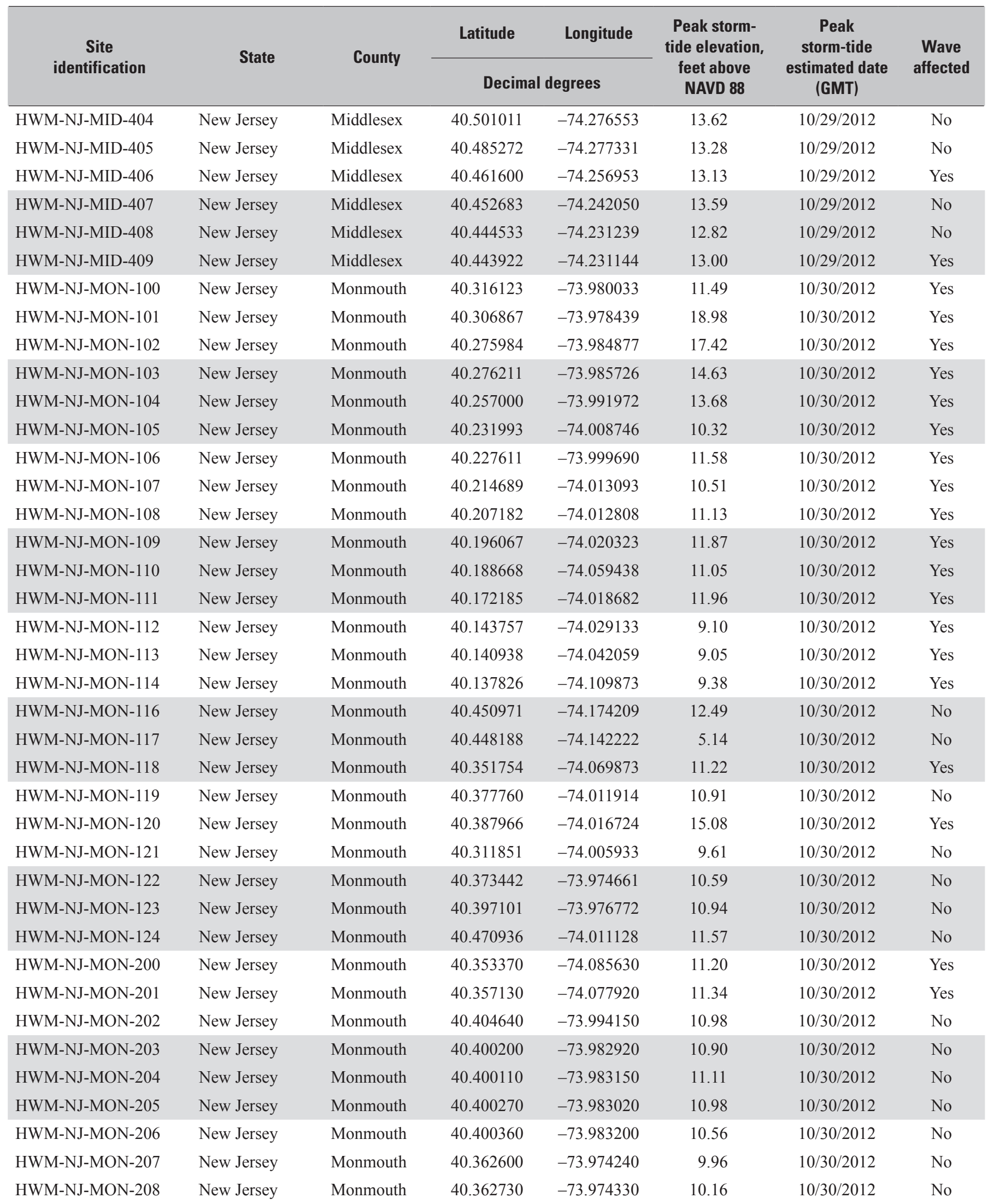


Table 6. Hurricane Sandy storm-tide high-water-mark data collected and surveyed by U.S. Geological Survey, by State.-Continued [NAVD 88, North American Vertical Datum of 1988; GMT, Greenwich Mean Time]

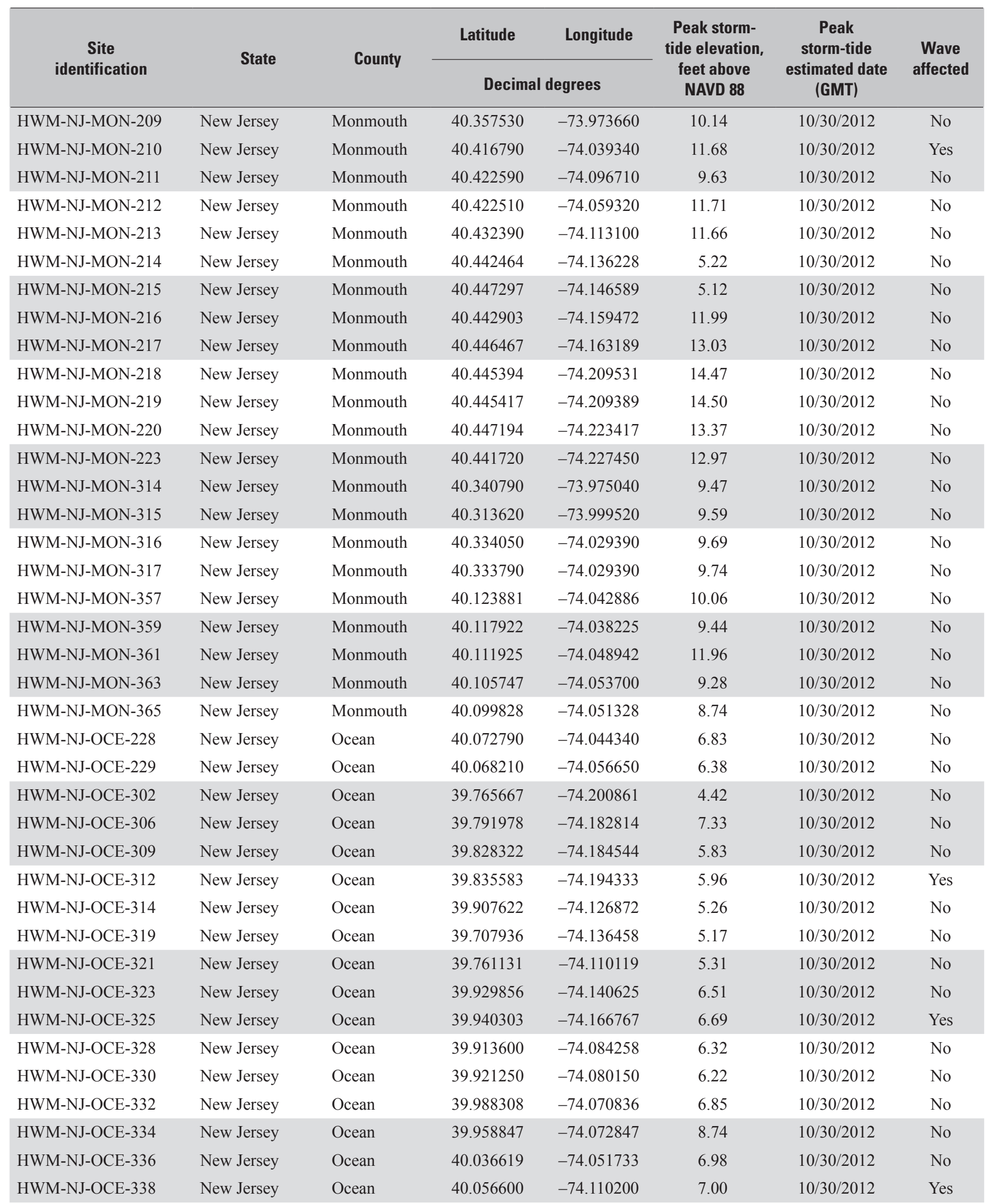


Table 6. Hurricane Sandy storm-tide high-water-mark data collected and surveyed by U.S. Geological Survey, by State.-Continued [NAVD 88, North American Vertical Datum of 1988; GMT, Greenwich Mean Time]

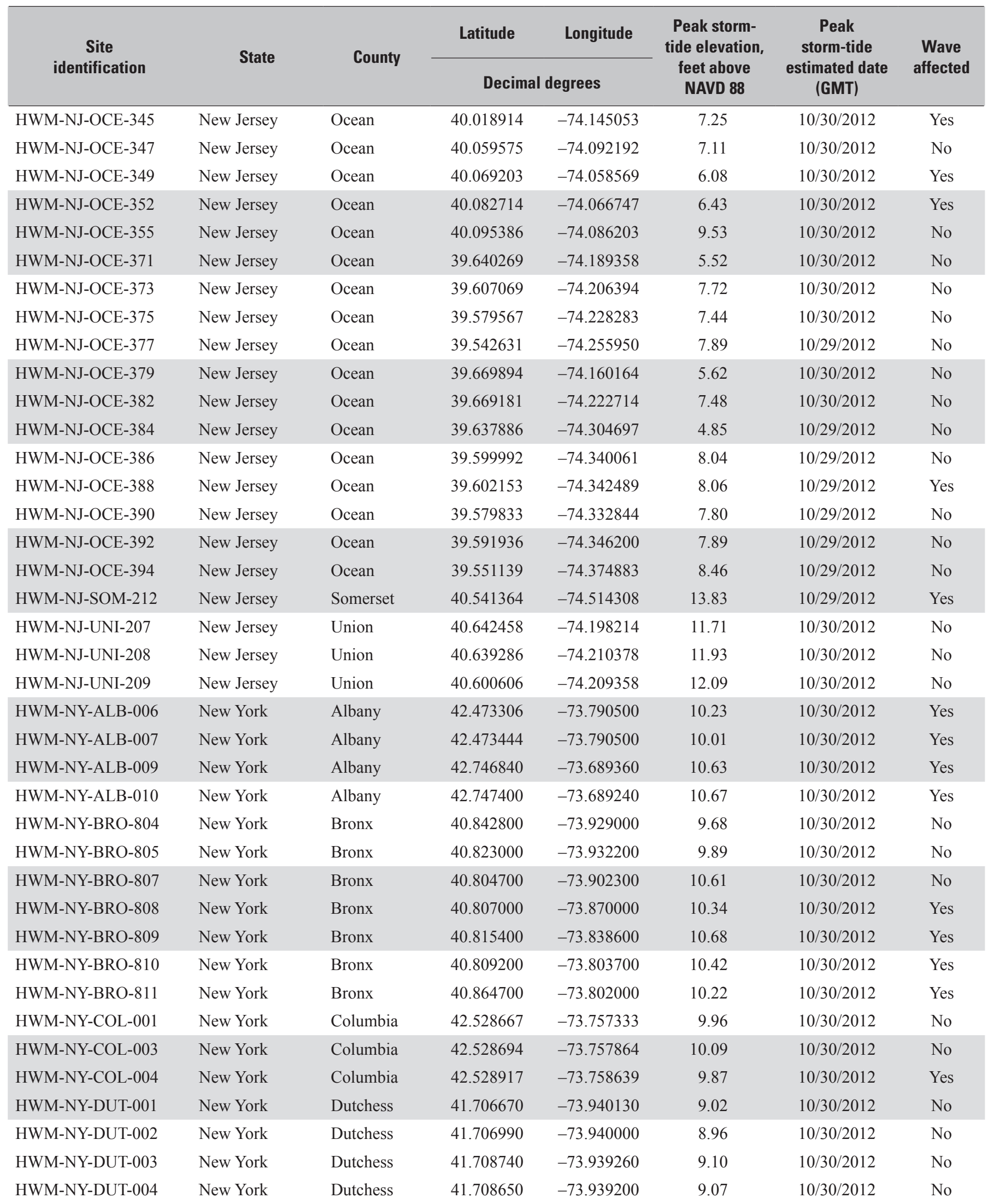


Table 6. Hurricane Sandy storm-tide high-water-mark data collected and surveyed by U.S. Geological Survey, by State.-Continued [NAVD 88, North American Vertical Datum of 1988; GMT, Greenwich Mean Time]

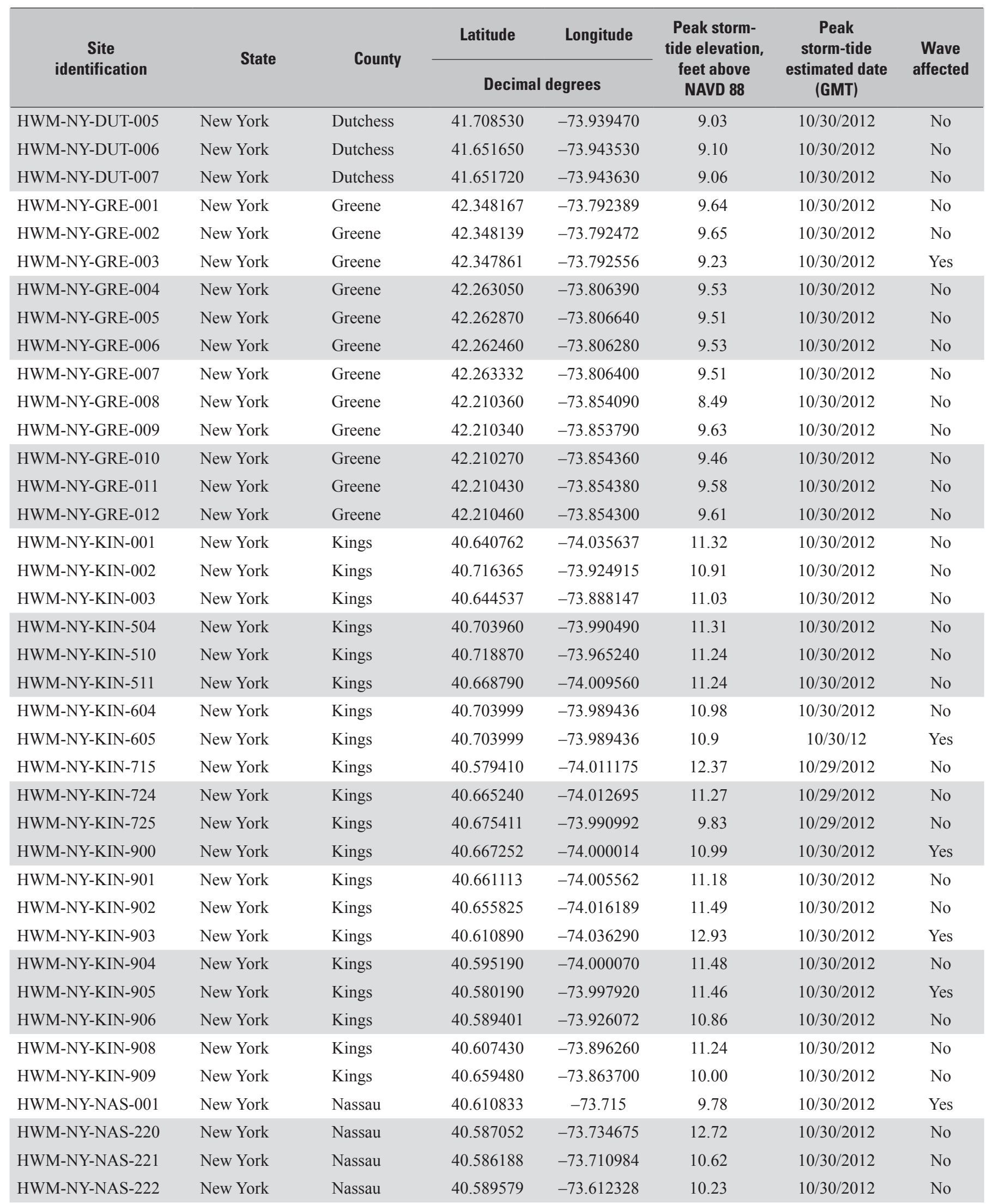


Table 6. Hurricane Sandy storm-tide high-water-mark data collected and surveyed by U.S. Geological Survey, by State.-Continued [NAVD 88, North American Vertical Datum of 1988; GMT, Greenwich Mean Time]

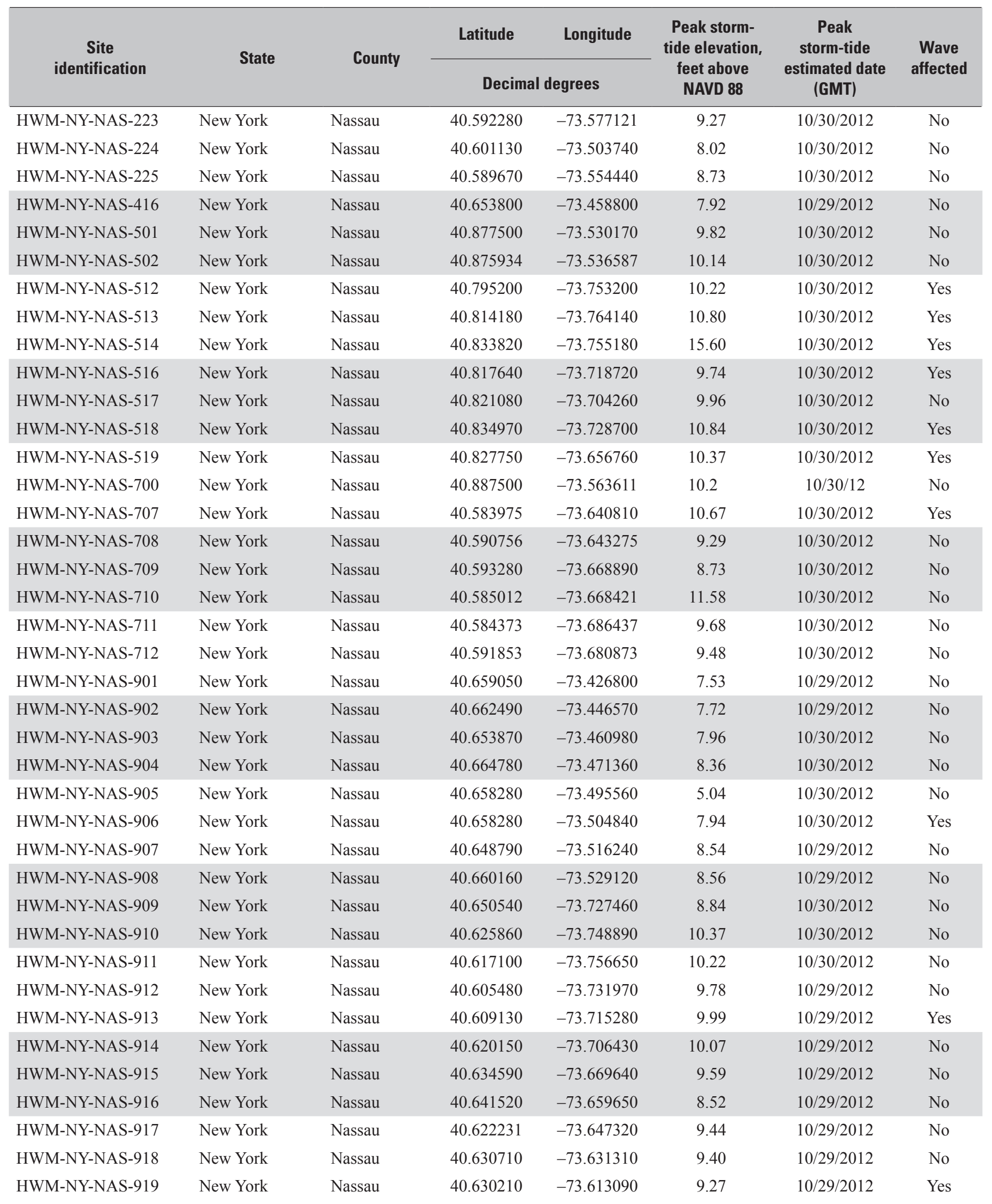


Table 6. Hurricane Sandy storm-tide high-water-mark data collected and surveyed by U.S. Geological Survey, by State.-Continued [NAVD 88, North American Vertical Datum of 1988; GMT, Greenwich Mean Time]

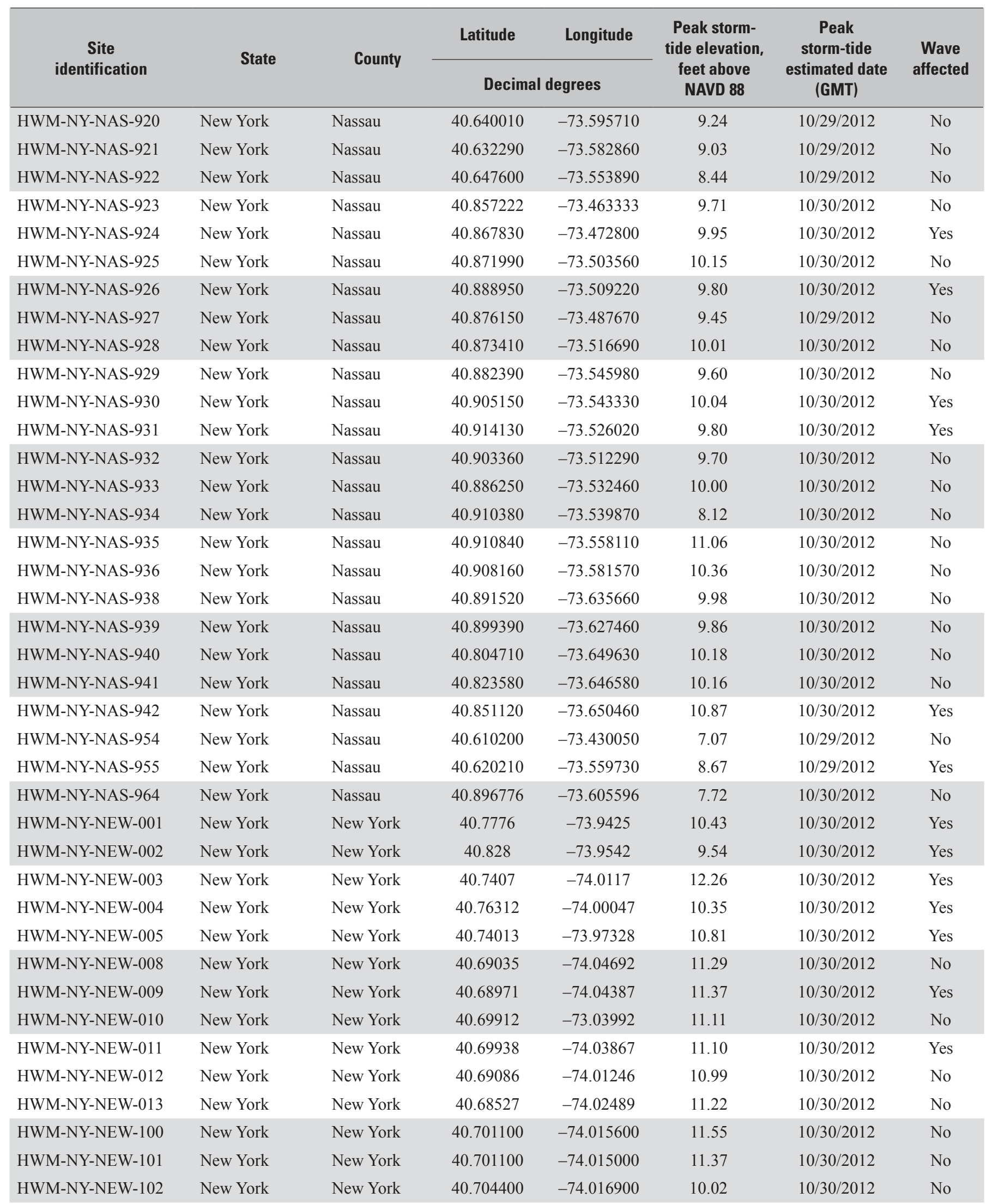


Table 6. Hurricane Sandy storm-tide high-water-mark data collected and surveyed by U.S. Geological Survey, by State.-Continued [NAVD 88, North American Vertical Datum of 1988; GMT, Greenwich Mean Time]

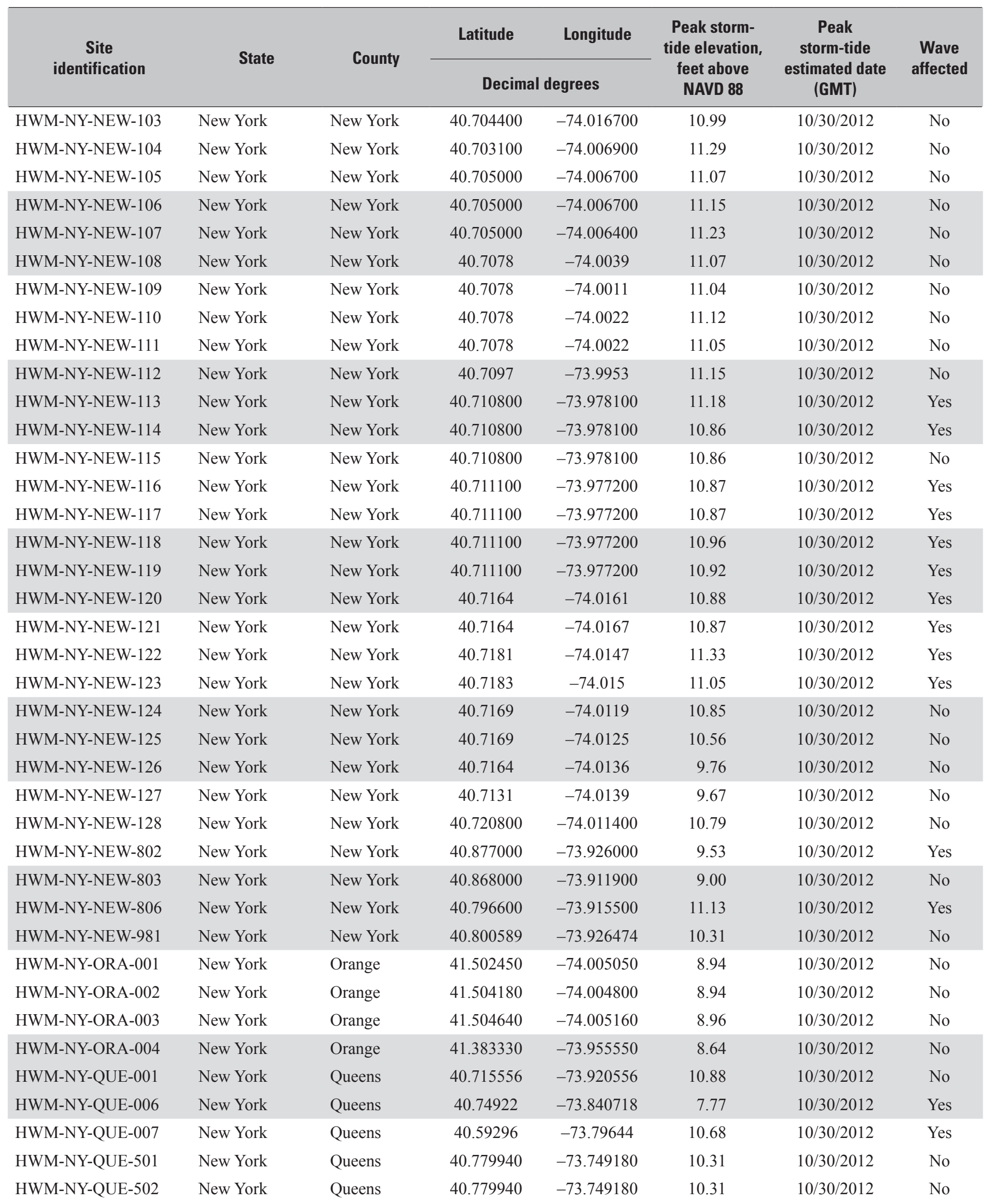


Table 6. Hurricane Sandy storm-tide high-water-mark data collected and surveyed by U.S. Geological Survey, by State.-Continued [NAVD 88, North American Vertical Datum of 1988; GMT, Greenwich Mean Time]

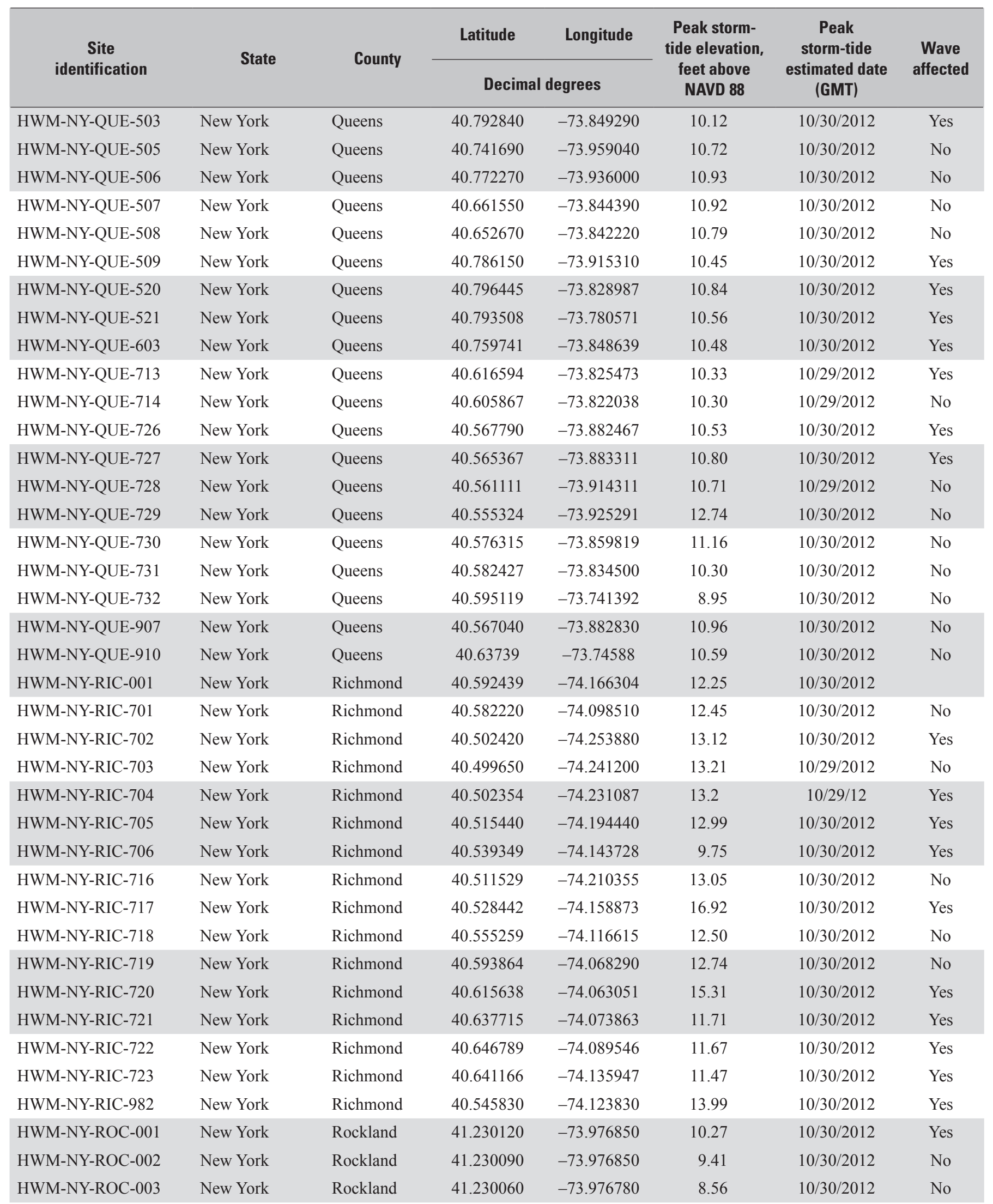


Table 6. Hurricane Sandy storm-tide high-water-mark data collected and surveyed by U.S. Geological Survey, by State.-Continued [NAVD 88, North American Vertical Datum of 1988; GMT, Greenwich Mean Time]

\begin{tabular}{|c|c|c|c|c|c|c|c|}
\hline \multirow{2}{*}{$\begin{array}{c}\text { Site } \\
\text { identification }\end{array}$} & \multirow{2}{*}{ State } & \multirow{2}{*}{ County } & Latitude & Longitude & \multirow{2}{*}{$\begin{array}{l}\text { Peak storm- } \\
\text { tide elevation, } \\
\text { feet above } \\
\text { NAVD } 88\end{array}$} & \multirow{2}{*}{$\begin{array}{c}\text { Peak } \\
\text { storm-tide } \\
\text { estimated date } \\
\text { (GMT) }\end{array}$} & \multirow{2}{*}{$\begin{array}{l}\text { Wave } \\
\text { affected }\end{array}$} \\
\hline & & & \multicolumn{2}{|c|}{ Decimal degrees } & & & \\
\hline HWM-NY-ROC-004 & New York & Rockland & 41.229900 & -73.976880 & 9.44 & $10 / 30 / 2012$ & No \\
\hline HWM-NY-ROC-005 & New York & Rockland & 41.229890 & -73.976970 & 11.12 & $10 / 30 / 2012$ & Yes \\
\hline HWM-NY-SUF-002 & New York & Suffolk & 41.073942 & -72.318901 & 6.21 & $10 / 30 / 2012$ & No \\
\hline HWM-NY-SUF-003 & New York & Suffolk & 40.9325 & -72.615556 & 7.22 & $10 / 30 / 2012$ & Yes \\
\hline HWM-NY-SUF-004 & New York & Suffolk & 40.747598 & -73.150391 & 6.10 & $10 / 30 / 2012$ & No \\
\hline HWM-NY-SUF-005 & New York & Suffolk & 40.965219 & -72.771869 & 9.1 & $10 / 29 / 12$ & Yes \\
\hline HWM-NY-SUF-302 & New York & Suffolk & 41.132470 & -72.258640 & 6.22 & $10 / 29 / 2012$ & No \\
\hline HWM-NY-SUF-303 & New York & Suffolk & 41.143330 & -72.312730 & 5.84 & $10 / 29 / 2012$ & Yes \\
\hline HWM-NY-SUF-304 & New York & Suffolk & 41.143310 & -72.312430 & 6.36 & $10 / 29 / 2012$ & Yes \\
\hline HWM-NY-SUF-305 & New York & Suffolk & 41.130700 & -72.328220 & 6.95 & $10 / 29 / 2012$ & No \\
\hline HWM-NY-SUF-306 & New York & Suffolk & 41.131180 & -72.328480 & 6.02 & $10 / 29 / 2012$ & No \\
\hline HWM-NY-SUF-307 & New York & Suffolk & 40.989800 & -72.470770 & 7.08 & $10 / 29 / 2012$ & No \\
\hline HWM-NY-SUF-308 & New York & Suffolk & 40.915930 & -72.637760 & 8.60 & $10 / 29 / 2012$ & Yes \\
\hline HWM-NY-SUF-401 & New York & Suffolk & 40.747500 & -72.854900 & 5.69 & $10 / 30 / 2012$ & No \\
\hline HWM-NY-SUF-408 & New York & Suffolk & 40.915000 & -72.661700 & 7.80 & $10 / 29 / 2012$ & No \\
\hline HWM-NY-SUF-409 & New York & Suffolk & 40.904200 & -72.619900 & 7.72 & $10 / 30 / 2012$ & No \\
\hline HWM-NY-SUF-410 & New York & Suffolk & 40.850400 & -72.503800 & 6.61 & $10 / 29 / 2012$ & No \\
\hline HWM-NY-SUF-411 & New York & Suffolk & 40.844800 & -72.572500 & 6.90 & $10 / 29 / 2012$ & No \\
\hline HWM-NY-SUF-412 & New York & Suffolk & 40.818900 & -72.624500 & 6.35 & $10 / 30 / 2012$ & No \\
\hline HWM-NY-SUF-413 & New York & Suffolk & 40.787700 & -72.749500 & 6.32 & $10 / 30 / 2012$ & No \\
\hline HWM-NY-SUF-415 & New York & Suffolk & 40.774100 & -72.816100 & 6.51 & $10 / 29 / 2012$ & No \\
\hline HWM-NY-SUF-417 & New York & Suffolk & 40.663000 & -73.413000 & 7.54 & $10 / 29 / 2012$ & No \\
\hline HWM-NY-SUF-418 & New York & Suffolk & 40.632134 & -73.216211 & 9.43 & $10 / 29 / 2012$ & No \\
\hline HWM-NY-SUF-419 & New York & Suffolk & 40.644000 & -73.157000 & 8.01 & $10 / 29 / 2012$ & No \\
\hline HWM-NY-SUF-420 & New York & Suffolk & 40.634550 & -73.203050 & 4.17 & $10 / 29 / 2012$ & No \\
\hline HWM-NY-SUF-421 & New York & Suffolk & 40.879800 & -72.449900 & 6.57 & $10 / 29 / 2012$ & No \\
\hline HWM-NY-SUF-422 & New York & Suffolk & 40.869000 & -72.392000 & 7.92 & $10 / 30 / 2012$ & No \\
\hline HWM-NY-SUF-424 & New York & Suffolk & 41.006300 & -72.035000 & 5.20 & $10 / 29 / 2012$ & No \\
\hline
\end{tabular}


Table 6. Hurricane Sandy storm-tide high-water-mark data collected and surveyed by U.S. Geological Survey, by State.-Continued [NAVD 88, North American Vertical Datum of 1988; GMT, Greenwich Mean Time]

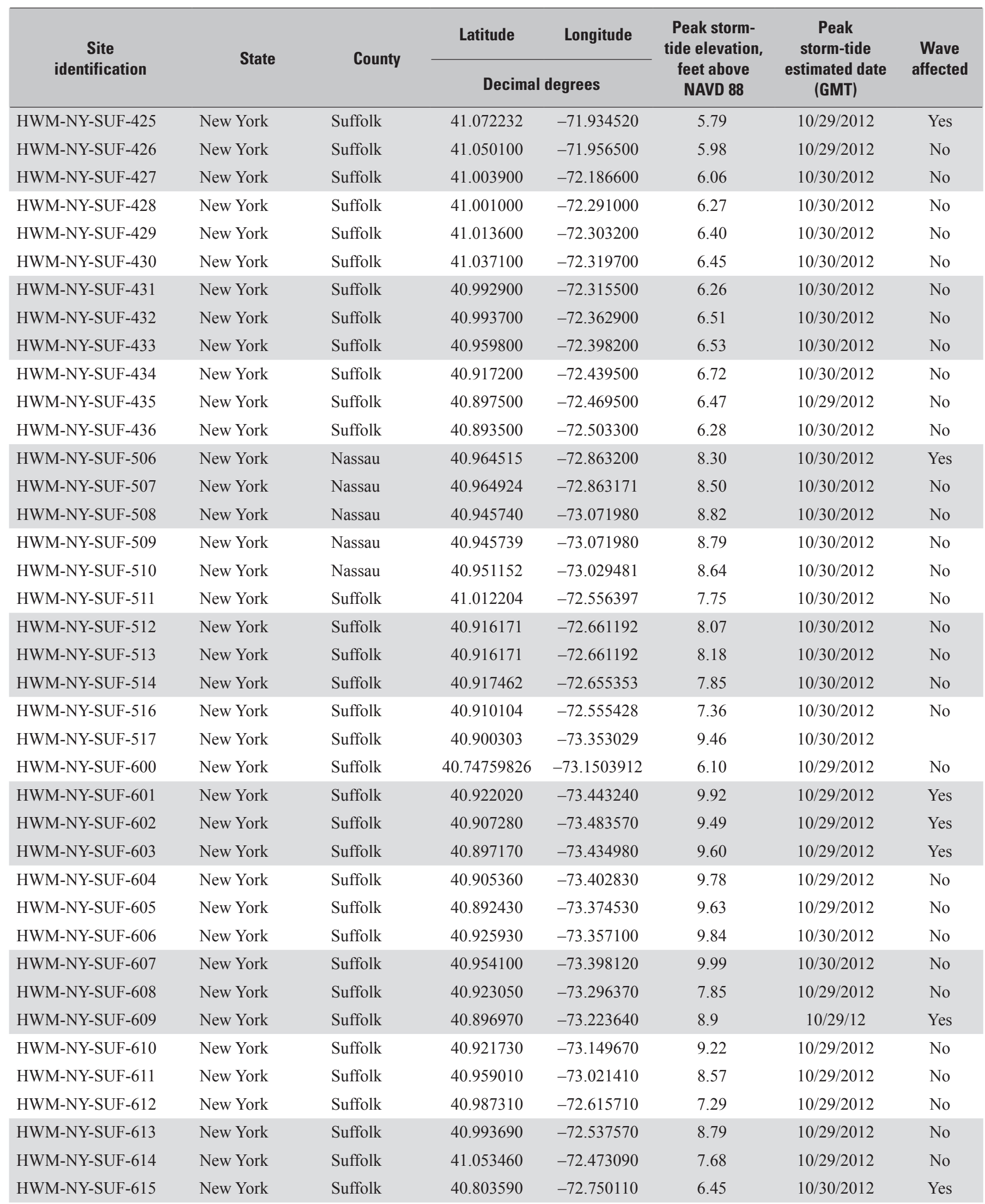


Table 6. Hurricane Sandy storm-tide high-water-mark data collected and surveyed by U.S. Geological Survey, by State.-Continued [NAVD 88, North American Vertical Datum of 1988; GMT, Greenwich Mean Time]

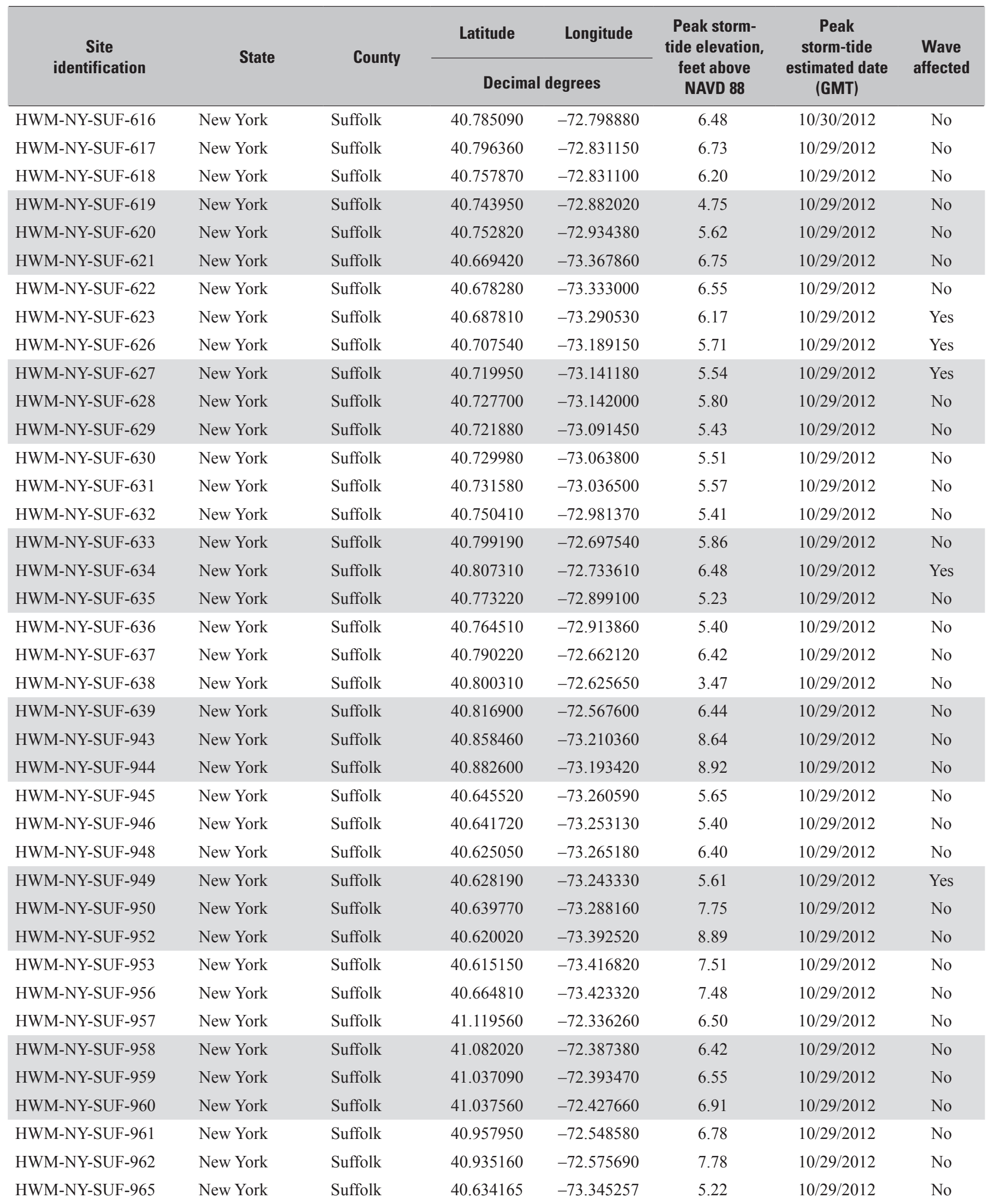


Table 6. Hurricane Sandy storm-tide high-water-mark data collected and surveyed by U.S. Geological Survey, by State.-Continued [NAVD 88, North American Vertical Datum of 1988; GMT, Greenwich Mean Time]

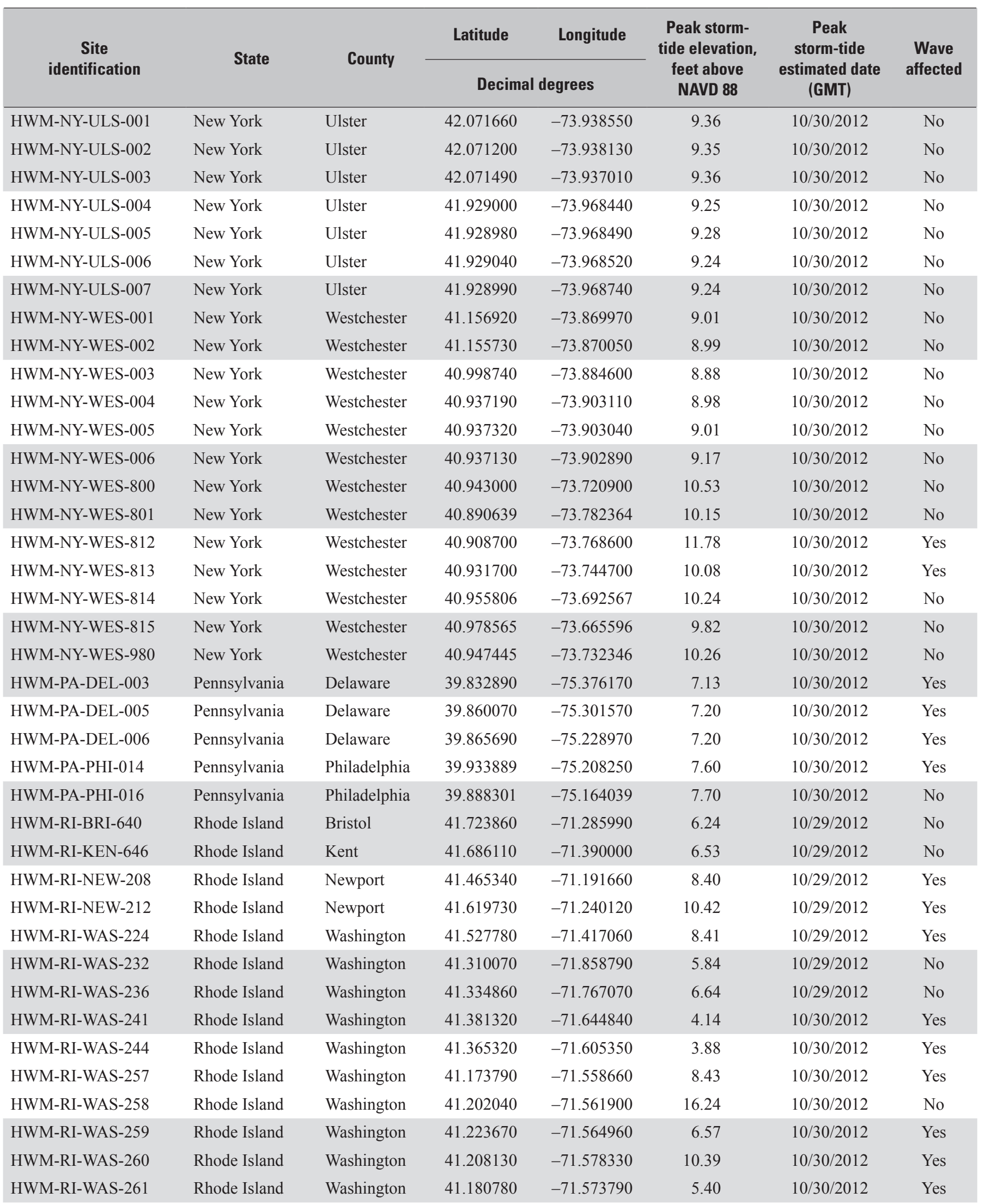


Table 6. Hurricane Sandy storm-tide high-water-mark data collected and surveyed by U.S. Geological Survey, by State.-Continued [NAVD 88, North American Vertical Datum of 1988; GMT, Greenwich Mean Time]

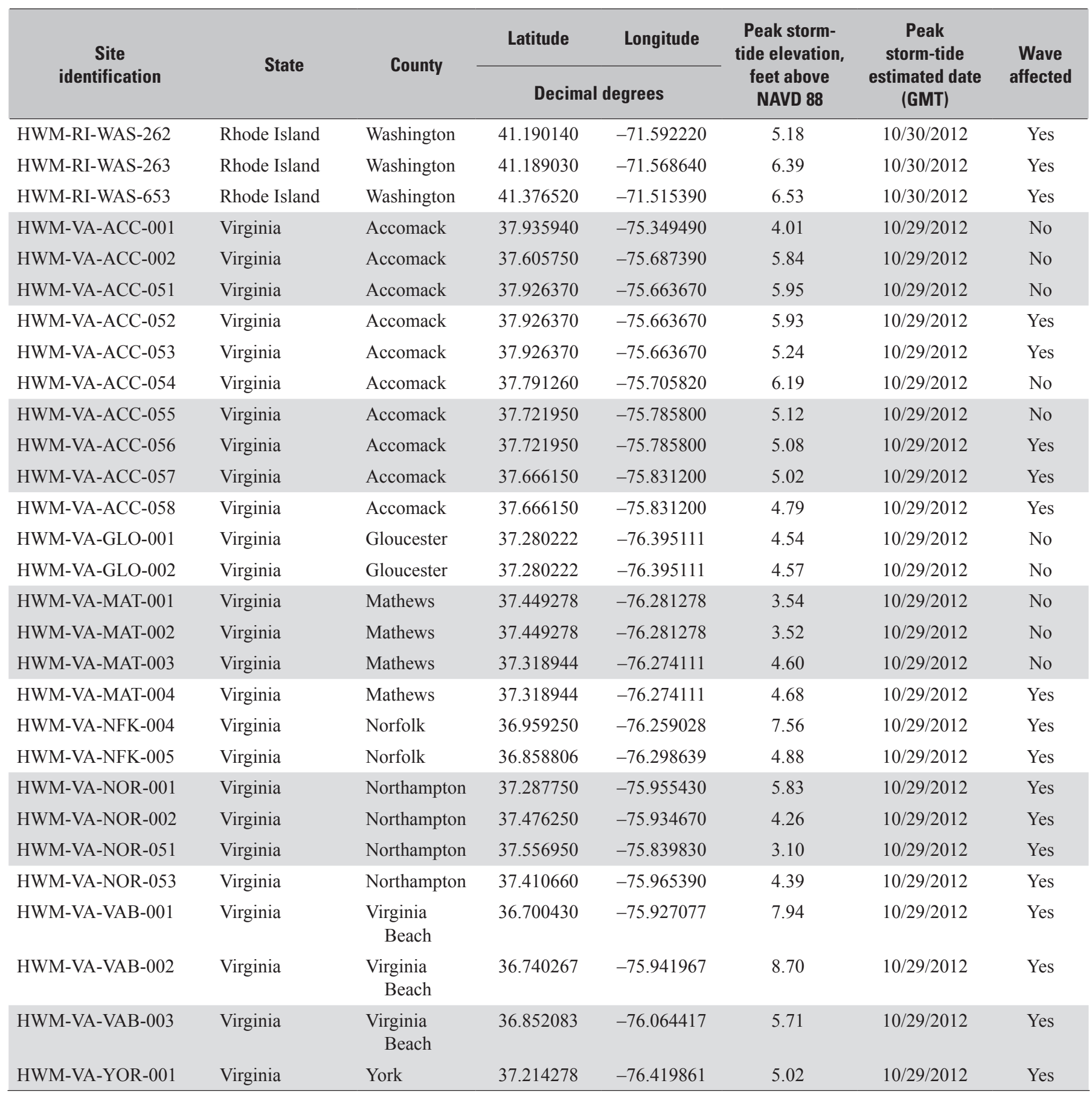




\section{Glossary}

EDT Eastern daylight time

EST Eastern standard time

FEMA Federal Emergency Management Agency

GMT Greenwich Mean Time

GNSS Global Navigation Satellite Systems

HWM High-water mark

NAVD 88 North American Vertical Datum of 1988

NGS National Geodetic Survey

NGVD 29 National Geodetic Vertical Datum of 1929

NOAA National Oceanic and Atmospheric Administration

RDG Rapid deployment gage

RTN Real-Time Network

storm surge Water level rise caused by a storm over and above the predicted astronomical tide

storm tide Water level rise due to the combination of storm surge and the astronomical tide

streamgage Instrumentation used to measure water level and corresponding streamflow

tide gage Instrumentation used to measure coastal water level

USGS U.S. Geological Survey 


\section{Conversion Factors and Datums}

\begin{tabular}{|c|c|c|}
\hline Multiply & By & To obtain \\
\hline \multicolumn{3}{|c|}{ Length } \\
\hline inch & 2.54 & centimeter $(\mathrm{cm})$ \\
\hline inch & 25.4 & millimeter (mm) \\
\hline foot $(\mathrm{ft})$ & 0.3048 & meter $(\mathrm{m})$ \\
\hline mile (mi) & 1.609 & kilometer (km) \\
\hline mile, nautical (nmi) & 1.852 & kilometer (km) \\
\hline yard (yd) & 0.9144 & meter $(\mathrm{m})$ \\
\hline \multicolumn{3}{|c|}{ Pressure } \\
\hline atmosphere, standard (atm) & 101.3 & kilopascal (kPa) \\
\hline bar & 100 & kilopascal (kPa) \\
\hline inch of mercury at $60^{\circ} \mathrm{F}$ (in $\mathrm{Hg}$ ) & 3.377 & kilopascal (kPa) \\
\hline $\begin{array}{l}\text { pound-force per square inch } \\
\qquad\left(\mathrm{lbf} / \mathrm{in}^{2}\right)\end{array}$ & 6.895 & kilopascal (kPa) \\
\hline pound per square foot $\left(\mathrm{lb} / \mathrm{ft}^{2}\right)$ & 0.04788 & kilopascal (kPa) \\
\hline pound per square inch $\left(\mathrm{lb} / \mathrm{in}^{2}\right)$ & 6.895 & kilopascal (kPa) \\
\hline
\end{tabular}

Temperature in degrees Celsius $\left({ }^{\circ} \mathrm{C}\right)$ may be converted to degrees Fahrenheit $\left({ }^{\circ} \mathrm{F}\right)$ as follows:

$$
{ }^{\circ} \mathrm{F}=\left(1.8 \times{ }^{\circ} \mathrm{C}\right)+32
$$

Temperature in degrees Fahrenheit $\left({ }^{\circ} \mathrm{F}\right)$ may be converted to degrees Celsius $\left({ }^{\circ} \mathrm{C}\right)$ as follows:

$$
{ }^{\circ} \mathrm{C}=\left({ }^{\circ} \mathrm{F}-32\right) / 1.8
$$

Vertical coordinate information is referenced to the North American Vertical Datum of 1988 (NAVD 88) unless otherwise noted.

Horizontal coordinate information is referenced to the North American Datum of 1983 (NAD 83) unless otherwise noted.

Elevation, as used in this report, refers to distance above the vertical datum. 
